九州大学学術情報リポジトリ

Kyushu University Institutional Repository

\title{
Molecular phylogenetic study in genus Hydra
}

\section{Kawaida, Hitomi}

Graduated School of Sciences, Kyushu University

Shimizu, Hiroshi

National Institute of Genetics of Japan

Fuj isawa, Toshitaka

National Institute of Genetics of Japan

Tachida, Hidenori

Department of Biology, Faculty of Sciences, Kyushu University

他

http://hdl. hand le. net/2324/25995

出版情報：Gene. 468 (1/2)，pp. 30-40，2010-11-15. Elsevier バージョン：

権利関係: (C) 2010 Elsevier B. V. 
Molecular phylogenetic study in genus Hydra

Hitomi Kawaida ${ }^{a}$, Hiroshi Shimizu ${ }^{\mathrm{b}}$, Toshitaka Fujisawa ${ }^{\mathrm{b}}$, Hidenori Tachida ${ }^{\mathrm{c}}$, and Yoshitaka Kobayakawa ${ }^{\mathrm{c} *}$.

${ }^{\mathrm{a}}$ Graduated School of Sciences, Kyushu University,

Hakozaki 6-10-1, Higashi-ku, Fukuoka, 812-8581, Japan

${ }^{\mathrm{b}}$ National Institute of Genetics of Japan,

Yata 1111, Mishima, 411-8540, Japan

${ }^{c}$ Department of Biology, Faculty of Sciences, Kyushu University.

Hakozaki 6-10-1, Higashi-ku, Fukuoka, 812-8581, Japan

*To whom all correspondences should be addressed.

E-mail Address: $\quad$ kbykwrcb@kyushu-u.org,

( $2^{\text {nd }}$ Address: kobayakawa.yoshitaka.890@m.kyushu-u.ac.jp)

Telephone \& Fax: +81926423901 


\begin{abstract}
Among 8000 - 9000 species of Cnidaria, only several dozens species of Hydrozoa have been found in the fresh water. Hydra is such a fresh water polyp and has been used as a good material for research in developmental biology, regeneration and pattern formation. Although the genus Hydra has only a few ten species, its distribution is cosmopolitan. The phylogenetic relationship between hydra species is fascinating from the aspect of evolutionary biology and biogeography. However, only a few molecular phylogenetic studies have been reported on hydra. Therefore, we conducted a molecular phylogenetic study of the genus Hydra based on mitochondrial and nuclear nucleotide sequences using a hydra collection that has been kept in the National Institute of Genetics (NIG) of Japan. The results support the idea that four species groups comprise the genus Hydra. Within the viridissima group (green hydra) and braueri group, genetic distances between strains were relatively large. In contrast, genetic distances between strains among the vulgaris and oligactis groups were small irrespective of their geographic distribution. The vulgaris group strains were classified at least (as far as our investigated samples) into three sub-groups, vulgaris sub-group, carnea sub-group, and $H$. sp. (K5\&K6) sub-group. All of the vulgaris sub-group and $H$. $s p$. (K5\&K6) sub-group strains were collected in Eurasia. The carnea sub-group strains in NIG collection were all collected in North America. A few newly collected samples in Japan, however, suggested belonging to the carnea sub-group according to the molecular phylogenic analysis. This suggests a trans-Pacific distribution of the carnea sub-group hydra.
\end{abstract}

Key Words: genus Hydra, molecular phylogeny, mitochondrial DNA, nuclear DNA 


\section{Introduction}

Hydra, a member of phylum Cnidaria, class Hydrozoa, order Hydroida, family Hydridae, is a fresh water polyp that has no medusa stage and usually reproduces by budding asexually. Because of its simple body plan and remarkable regeneration activity, hydra has been used as one of the best model animal in developmental biology, especially in study on pattern formation (Bosch and Fujisawa, 2001; Bosch and Khalturin, 2002; Steele, 2002; Holstein et al, 2003) and on the stem cell system (Bosch, 2009; Bosch et al., 2010).

Most of eumetazoans have bilateral symmetric body that consist of three germ layers and are referred as Bilateria. In the other eumetazoans, the Cnidaria is the largest and most diverse group. Its body wall consists of only two germ layers, ectoderm and endoderm. In Cnidaria, Anthozoa species have bilateral symmetry in the internal morphology whereas Medusozoa (Scyphozoa, Cubozoa, and Hydrozoa) species have radial or tetra radial symmetry body plan (Nielsen, 2001). Therefore cnidarian has been attracting interest from researchers in animal phylogeny and evolutionary biology, especially from the aspect of origin of the body plans in animals.

The Cnidaria comprise 8,000 - 9000 living species (Tudge, 2000), which occur mostly in marine aquatic habitats. Only several dozens species occur in fresh water habitats. (Dumont 1994, Jankowski, Collins and Campbell, 2008). Most of the fresh water cnidarian belong to the Hydrozoa, fresh water medusae (genus Limnocnida and Craspedacusta) and solitary polyp, hydra (genus Hydra). It is not unclear whether all freshwater cnidarians have a same origin or not. In the reported cnidarian molecular phylogenetic studies, however, species of hydra always formed a monophyletic group (Bridge et al, 1995; Collins, 2000 \& 2002; Collins et al., 2006). These suggest that an ancestor of hydra had made its way into fresh water and later that ancestor speciated into additional species. Although only a few phylogenetic studies on species in the genus Hydra have been reported, Campbell (1983, '87 and '89) proposed that genus Hydra consists of four species groups, viridissima group, braueri group, vulgaris group, and oligactis group, based mainly on the morphological characters. Jankowski et al. (2008) suggested that the viridissima group and the vulgaris group were probably present before the continents separated. They also suggested that the oligactis group and the braueri groups are restricted to the northern continents and presumably arose after the separation of northern and southern landmasses. In this manner, the origin of fresh 
water cnidarians and their diversity across all continents pose intriguing problems. To solve these problems, molecular phylogenetic studies of freshwater cnidarians are indispensable.

Only a few molecular phylogenetic studies, however, have been reported. Thomsen and Bosch (2006) reported differences in gene number and structure within the PPOD (foot-specific peroxidase) gene family in hydra species. Their results supported Campbell's idea of four groups and also suggested unexpected genomic variation within closely related species of hydra. Hemmrich et al. (2007) estimated the phylogenetic relations with molecular data from two nuclear (18S rDNA, 28S rDNA) and two mitochondrial (16S rRNA, cytochrome oxidase subunit I (COI)) genes among eight members of the genus Hydra, which have been kept in laboratories and often used for many researches. Their results also supported Campbell's idea; genus Hydra consists of four species groups, viridissima group, braueri group, vulgaris group, and oligactis group. Using molecular phylogenetic analyses, they found that in contrast to its initial description, the strain, Hydra vulgaris (strain AEP), used for making transgenic hydra (Wittlieb et al., 2006), is more closely related to $H$. carnea than to the other species of hydra and stated the difficulty of identifying hydra species based only on the morphological characters. Therefore, molecular phylogenetic studies on the genus Hydra are eagerly anticipated, as they will provide basic data for diverse studies on hydra.

Mitochondrial DNA (MtDNA) has been used extensively for the molecular phylogenetic analysis in a wide range of organisms. MtDNA in Medusozoa (Schiphozoa, Cubozoa, Hydrozoa) displays in the form of a linear molecule (Bridge, Schierwater, DeSalle, Buss, 1992) while most of organisms, including the Anthozoa, have circular form MtDNA. In two species of hydra, H. oligactis and $H$. maganipapillata, mitochondrial DNA sequences have been determined recently (Kayal and Lavrov, 2008; Voigt, Erpenbeck and Wörheide, 2008). They showed that $H$. oligactis has one linear MtDNA and H. maganipapillata has two fragments of linear MtDNA. Therefore, analyzing MtDNA in hydra species is intriguing with the both object of phylogeny of hydra and evolution of mitochondrial DNA itself.

National Institute of Genetics Japan maintains a hydra strain collection that includes several dozens strains (identified as several species). Using this collection and some newly collected hydra specimens in Japan, we studied molecular phylogenies in 
the genus Hydra with molecular data from mitochondrial (lrRNA, tRNA, cytochrome oxidase subunit II; COII, and cytochrome oxidase subunit I; COI) DNAs, and two nuclear genes (CnNOS1, EFla).

\section{Materials and methods}

2.1. Hydra strains and out groups for analysis

Molecular phylogenetic analyses were carried out with several ten hydra strains that have been kept in the National Institute of Genetics (NIG) of Japan and some specimens collected in Fukuoka prefecture in Japan.

All H. magnipapillata strains (A1;nem-3, A9;sf-1, B4, B8;105, B10;identified as H. japonica, B11, B12, C2, D1;mini-1, D5;nem-11, D7;maxi-1, E4, E6, F2;reg16, J1, J2, J6, J7, J10) had been collected in Japan. H. vulgaris K9 strain originated in Europe and AEP, and $\underline{\mathrm{M} 5}$ strains originated in USA. Hydra attenuata (B6, K5 $, \underline{\mathrm{K} 6}, \mathrm{~K} 7, \mathrm{~L} 2)$ originated in Europe and H. carnea (L4) in USA. Hydra oligactis G7 strain originated in Germany and M2, M4 strains in USA. Hydra fusca (K12) was of European origin. Pelmatohydra robusta (Japanese oligactis group hydra, B1, B2, B3, L5) had been collected in Japan. Hydra circumcincta strains (K8, M7) originated in Europe and $H$. utahensis (L10) and H. hymanae (M1) in USA. Some of $H$. viridissima \& viridis strains (B5, K10, M9, M10, N11) originated in Europe and L9, M8 strains in USA. Sampling location of Hydra sp. (J8) has been unknown. All these strains have been kept at NIG and were used for the molecular phylogenetic analyses in this study. Species identification of the underlined strains, K5, K6, M2, and M5, were suggested to be inaccurate as discussed later in this report. Therefore, we referred these strains as Hydra $s p$. in some cases.

The polyps were cultured under the standard condition. Each strain was kept in a plastic dish filled with a hydra culture solution $(1 \mathrm{mM} \mathrm{NaCl}, 1 \mathrm{mM} \mathrm{CaCl} 2,0.1 \mathrm{mM}$ $\mathrm{KCl}, 0.1 \mathrm{mM} \mathrm{MgS0}$, $1 \mathrm{mM}$ tris-(hydroxymethyl)-aminomethane; $\mathrm{pH}$ 7.4, adjusted with $\mathrm{HCl}$ ) at $20^{\circ} \mathrm{C}$. The polyps were fed with newly hatched nauplii of Artemia two times a week.

We collected polyps in 5 ponds at 4 locations, , Tanushimaru, Kasuga, Tajima, and Ohori (FT 08, KAS 20, ONI 5, FO1 G, FO2 03, FO2 05), in Fukuoka prefecture in Japan. All polyps collected were identified as belonging to the vulgaris group species 
based on morphological traits by the stereoscopic microscopy observation. These several dozens polyps were also used for the molecular phylogenetic analyses. FT 08 represented 4 samples that had been collected at the same location and showed the same nucleotide sequence. Similarly, KAS 20, ONI 5, FO1 G, FO2 03, and FO2 05 represented 12, 6, 12, 3, and 3 samples respectively.

The NCBI genomic data of marine hydrozoans Anthozoa Nematostella vectensis (DQ643835, AY730693, AB126336) served as out groups in the phylogenetic analyses.

2.2. DNA isolation, PCR amplification and sequencing

Genomic DNA and mitochondrial DNA from each strain was isolated using a genomic DNA extraction Kit (DNeasy Tissue Kit, QIAGEN)). To obtain a DNA template for the PCR amplification from one polyp, a polyp was homogenized in a 50 $\mu \mathrm{l} \mathrm{PCR}$ buffer (50mM KCl, 10mM tris-hydroxymethyl-aminomethane $\mathrm{HCl} \mathrm{pH} 8.3$, $0.1 \% \mathrm{NP}-40$ ), treated with proteinase $\mathrm{K}$ (add $2 \mu 120 \mathrm{mg} / \mathrm{ml}$ proteinase $\mathrm{K}$ stock solution, Takara Bio Inc.) at $55^{\circ} \mathrm{C}$ for one hour and then incubated at $95^{\circ} \mathrm{C}$ for 15 minutes to inactivate the proteinase $\mathrm{K}$. The target DNA fragments (partial sequences of mitochondrial genes and some nuclear genes) were PCR amplified using degenerate primers and heterologous primers designed for each genes based on the MtDNA sequence of $H$. magnipapillata (GenBank Accession \#AF100773.1), CnNOS1 sequence of $H$. magnipapillata (GenBank Accession \#AB037080.1), and EF1a sequence of $H$. magnipapillata (GenBank Accession \#D79977.1). Primer sequences were as follows. For MtDNA IrRNA-tRNA(W)-COII locus, MtF 5'GTGAAAAACTAATCGAGTTAAGAGA -3' and MtR 5'CCACATAATTCTGAACATTGACC -3' were used. For MtDNA COI, G-COIF 5'TGGTGCATTTTCTGGAATGATAGGTAC -3', G-COIR 5'CTATCAGTTAGTAGCATAGTTATAGC -3' (for viridissima group), B-COIF 5' CTGGCATTACTAAAAAAAATAT -3', and B-COIR 5'AATATCATTACAAAAGCATGAGC -3' (for other groups) were used. For nuclear DNA, Nos1F 5' - AGTCAATTTTCTGTATTTCGTGA -3', Nos1R 5'TGTTTTCGGTTTTGGAGTAT -3', EF1aF 5' - ATTTACAAATGTGGTGGTATCG -3', and EF1aR 5'- CCAATTTTGTAGACATCTTGAAG -3' were used. 
The PCR amplified template DNA fragments were purified with Wizard SV Gel and PCR Clean-Up System (Promega). Sequence reactions were performed using BigDye Terminator v3.1 Cycle Sequencing Kit (Applied Biosystems). Samples were sequenced with ABI3100 Avant Genetic Analyzer (Applied Biosystems). All sequences have been submitted to DDBJ (Accession Nos. CnNOS1: AB565041-AB565065, EF1a: AB565066-AB565088, COI: AB565089- AB565143, lrRNA-tryptophan (W) tRNA-COII: AB565144 - AB565197).

\subsection{Phylogenetic Analysis}

Sequence alignments were generated using ClustalX (Thompson et al., 1997) or ClustalW (Thompson et al., 1994) included in MEGA4 soft ware package (Tamura et al., 2007; Kumar et al., 2008) or SeaView4.2 (Galtier et al., 1996). Alignments were optimized by hand using SeaView4.2 or MEGA4. Large insertions observed in MtDNA of L10 and M1 strains were deleted from the alignment. We used neighbor-joining (NJ), maximum parsimony (MP), maximum likelihood (ML), and Bayesian inference (BI) methods to infer phylogenetic relationships among the hydra species and strains.

For the NJ method, evolutionary distances were computed using the Nei-Gojobori method (Nei and Gojobori, 1986) and are in the units of the number of synonymous differences per synonymous site for nuclear DNA (CnNOS1, EF 1a genes). All positions containing alignment gaps and missing data were eliminated only in pairwise sequence comparisons. There were a total of 188 positions in the final dataset of CnNOS1 and of 204 positions in EFla. For mitochondrial DNA the p-distances were used because these sequences included more than a half-length non amino acid sequences coding region, lrRNA and tRNA(W). All positions containing alignment gaps and missing data were eliminated only in pairwise sequence comparisons. There were a total of 2439 positions in the final dataset. The evolutionary history was inferred using the NJ method (Saitou and Mei, 1987). Bootstrap consensus trees inferred from 10000 replicates were taken to represent the evolutionary history of the taxa analyzed (Felsenstein, 1985). We used MEGA4 for these analyses including the tree drawing.

We also used the MEGA4 for the maximum parsimony analyses. The MP tree was obtained using the Close-Neighbor-Interchange algorithm (Nei and Kumar, 2000) with search level 3 in which the initial trees were obtained with the random addition of sequences (10 replicates). All alignment gaps were treated as missing data. 
There were a total of 574 positions in the final dataset, out of which 161 were parsimony informative for CnNOS1 and a total of 612 positions in the final dataset, out of which 116 were parsimony informative for EFla. For mitochondrial DNA, there were a total of 2439 positions in the final dataset, out of which 745 were parsimony informative. In supplemental Figures, most parsimonious trees are shown with the percentage of replicate trees in which the associated taxa clustered together in the bootstrap test (1000 replicates).

PhyML (Guindon and Gascuel, 2003) was executed using the GTR (General Time Reversible) model through SeaView4.2 for the maximum likelihood analyses. For selecting models we used jModelTest (Posada, 2008). Starting trees were made by BioNJ. For tree searching operation 'best of NNI (nearest-neighbor interchange) and SPR (subtree pruning and regrafting)' was applied. Bootstrap values were calculated (1000 replicates).

Using Mr. Bayes v. 3.12 (Huelsenbeck and Ronquist, 2001), we executed Bayesian inference analyses. GTR model and sets across-site rate variation for gamma distribution with a proportion of invariant sites were applied. We ran the analysis for $500,000-1000,000$ generations and recorded the current tree and parameter values every 100 generations.

MEGA4 was also used to estimate synonymous substitution rates in the protein coding regions of mitochondrial DNA (COI, COII genes) and nuclear DNA (CnNOS1, EFla genes). We used the Nei-Gojobori method (1986) for the estimation.

\section{Results}

3.1 Molecular phylogenetic analyses between species in genus Hydra using mitochondrial genes

Figure 1 shows a phylogenetic tree drawn based on the ML analysis on the

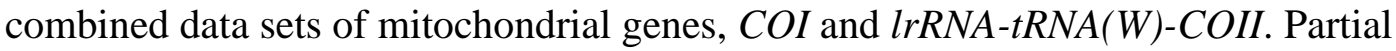
sequences of COI gene (459 nucleotides) and partial sequences of COII gene (606 nucleotides) were in the amino acid sequence coding regions. This tree was drawn with corresponding sequences of Nematostella vectensis as an out-group. 
Strains of $H$. viridissima and $H$ viridis (B5, K10, L9, M8, M9, M10, N11) and Hydra sp. (J8), which possessed symbiont green algae in their endodermal epithelial cells, formed a clade and it was sister to all other strains. In both phylogenetic trees drawn by NJ and MP analyses (see Suppl. Fig.1A, B), these viridissima group strains also formed a monophyletic group sister to all other strains of hydra species. Only the result of BI analysis suggested that braueri group and viridissima group made a monophyletic clade (Supple. Fig. 1C). However, the posterior probability of this clade was not so high, 0.56 .

All other strains diverged into three clades by the ML analysis (Fig. 1) and also by the NJ, MP, BI analyses (see Supple. Fig.1A, B, C). One of the clades consisted of the braueri group species (H. circumcincta; K8, M7, H. utahensis; L10, H. hymanae; M1). Another two clades were formed from the oligactis group (H. oligactis; G7, M4, $H$. fusca; K12, Pelmatohydra robusta; B1, B2, B3, L5, P) and the vulgaris group (H. magnipapillata; A1, A9, B4, B8, B10, B11, B12, C2, D1, D5, D7, E4, E6, F2, J1, J2, J6, J7, J10, H. vulgaris; K9, AEP, M5, H. attenuata; B6, K5, K6, K7, L2, H. carnea; L4, H. sp.; M2 and newly collected samples in Fukuoka, Japan; FO2 05, ONI 5, FO1 G, KAS20, FT 08, FO2 03), respectively. In the ML,NJ, MP, and BI analyses, the oligactis group strains and the vulgaris group strains formed a monophyletic clade (Fig.1 and Supple. Fig.1A, B, C) sister to the braueri group.

Figure 1 shows another distinct feature that the vulgaris group diverged into three sub-clades with very high bootstrap probabilities. One of them consisted of K5 and K6 strains that had been identified as H. attenuata in the NIG collection. Another clade consisted of L4 (had been identified as H. carnea), M5, AEP (had been identified as $H$. vulgaris), M2 (had been identified as $H$. oligactis) and newly collected samples (KAS 20, FT 08, FO2 03). All other strains of vulgaris group (H. magnipapillata; A1, A9, B4, B8, B10, B11, B12, C2, D1, D5, D7, E4, E6, F2, J1, J2, J6, J7, J10, H. vulgaris; K9, H. attenuata; B6, K7, L2, H. sp.; M2 and newly collected samples; ONI 5, FO1 G, FO2 05) formed still another clade (Fig.1). These three sub-clades were also recognized in NJ, MP, and BI trees (see Supple. Fig.1A, B, C). We named expediently these three sub-groups in vulgaris group, $H$. $s p(K 5, K 6)$ sub-group, carnea sub-group, and vulgaris sub-group, respectively.

These molecular phylogenetic analyses also suggested that genetic distances between strains within the vulgaris group or within each sub-group were very small 
irrespective of the geographic distribution of the used samples, Japan, Europe and North America. However, genetic distances between strains within the braueri group and viridissima group were not so small unlike the case of the vulgaris group.

3.2 Molecular phylogenetic analyses using nuclear DNAs, CnNOS1 and EFla, confirmed the inferred phylogenetic relationships in the genus Hydra based on the mitochondrial genes

We reconfirmed that hydra species clustered into the four monophyletic groups, the viridissima group, braueri group, oligactis group and vulgaris group based on the nuclear DNA sequence data analyzed by ML, NJ, and MP methods (Fig. 2, 3 and Supple. Fig. 2A, 2B, 3A, 3B). Only the result of BI analysis suggested viridissima group branched off into two clades (Supple. Fig. 2C, 3C). However, monophyly of the other three groups against viridissima group were also suggested by BI analysis as is the cases with ML, NJ, and MP analyses.

We also reaffirmed that the vulgaris group diverged into the three sub-groups, the vulgaris sub-group, carnea sub-group, and $H$. sp. $(K 5, K 6)$ sub-groups (Fig. 2, 3 and Supple. Fig. 2A - C, 3A - C).

3.3 Occurrence of large insertions of mitochondrial DNA has been presumed in hydra species among braueri group

In the course of sequencing the mitochondrial DNA, we found large insertions in the braueri group hydra species. In Hydra utahensis (L10), a 1297 bp insertion was found between the $\operatorname{lr} R N A$ coding region and the $t R N A(W)$ - COII coding region. $72 \mathrm{bp}$ sequence in anterior part of this insertion showed $82 \%$ identity with the $t R N A(W)$ region. Following it, from the 77 th nucleotide sequence position a 1200bp open reading frame (ORF) was recognized. Anterior part of 47 amino acid sequence of predicted translated product of this ORF showed $57 \%$ identity and $68 \%$ similarity with the COII amino acid sequence. Many fragments in the following part also showed similarity with parts of the COII (Supple. Fig. 4).

In Hydra hymanae (M1), behind the 9th nucleotide of the COII coding region, a 500 bp insertion was observed. However, this insertion did not have any large ORF and we could not fined any homology or similarity with known sequences. 


\subsection{Morphological aspects of K5 and K6 strains}

In vulgaris group, both the vulgaris sub-group and the carnea sub-group consisted of well known strains, such as a standard wild type strain Hydra magnipapillata 105 (B8) whose whole genomic sequence has been determined, strain AEP which was one of the most important strain for the future research because of its usefulness in transgenic hydra production. Polyps between these two sub-groups have shared common morphological traits, tentacles' length being longer than the body length, characteristic size and shape of nematocysts, holotrichous isorhizas with narrowly oval shape and transverse coils in the upper half.

K5 and K6 strains have been identified as Hydra attenuata along with B6, K7, and L2 strains in the NIG stock list. The B6, K7, L2 strains belonged the vulgaris sub-group in the present molecular phylogenetic analyses (Fig. 1 and Supple. Fig.1A, B, C), and their morphological traits resemble those of the vulgaris and carnea sub-group strains. K5 and K6 strains, however, have similar or shorter tentacles' length compared with the body length (Fig. 4A). The characteristics of holotrichous isorhizas with narrowly oval shape and transverse coils in the upper half are common with the other vulgaris sub-groups. However, the width of the holotrichous isorhiza was nearly half of the length, and the horizontal coils have only a few turns. Relatively larger size of desmoneme was another specific character. Its length was longer than atrichous isorhiza and similar to that of holotrichous isorhiza (Fig. 5). These morphological traits of K5 and K6 strains support independently the sub-group clustering of these strains in the vulgaris group along with the results of molecular phylogenetic analyses (Fig. $1-3$ and Supple. Fig. 1 - 3).

3.5 Substitution rate difference between mitochondrial DNA and nuclear DNA in hydra Using the $C O I$ and $C O I I$ nucleotide sequence as representatives of mitochondrial genes, we estimated synonymous substitution rates in some of the hydra strains. For the estimation, we used the modified Nei-Gojobori method with the Jukes-Cantor correction. Partial nucleotide sequences of CnNOS1 and EF1a were used as nuclear gene representatives. We compared the synonymous substitution rate between the MtDNA (COI, COII) and nuclear DNA (CnNOS1, EFla) among several strains of hydra (Table 1). Both substitutions in the mitochondrial genes and nuclear genes between groups of hydra (viridissima, braueri, oligactis and vulgaris groups) 
were considered already saturated. In contrast, between closely related strains such as between M9 - M10 and K8 - M7, almost no substitutions occurred,. Therefore, we compared the substitution rates between strains belonging to the same group but not so closely related to each other, such as L4 and L2 in the vulgaris group, and G7 and B3 in the oligactis group. Our results suggested that the substitution rate in the mitochondrial genome was 2.4-7.8 times higher than that of the nuclear genome in hydra (Table 1).

\section{Discussion}

4.1 Genus Hydra consist of four distinct species groups

Campbell (1987) suggested that hydras fit naturally into four clusters of species termed, the oligactis, vulgaris, viridissima, and braueri groups, based on several phenotypic characters. According to Campbell's keys (1983, 1987), species of the viridissima group are characterized by intracellular algal symbiont in the endodermal epithelium and small stenotele nematocysts not longer than $11 \mu \mathrm{m}$ (Fig. 5). Taxonomic keys to the braueri group members are plump (at least half as broad as long) holotrichous isorhiza (Fig. 5) and embryo theca without spines. The oligactis group is characterized by their buds that acquire 2 lateral tentacles before other tentacles appear; otherwise, stenotele nematocysts at least 1.5 times as long as its width. Holotrichous isorhiza without transverse coils (Fig. 5) is also a trait of the oligactis group species except for Hydra pseudoligactis (=Hydra canadensis). The vulgaris group is characterized by its buds acquiring tentacles almost synchronously; otherwise, stenotele nematocysts less than 1.5 times as long as its width. Holotrichous isorhiza with transverse coils and slender shape is also a trait of the vulgaris group.

All strains of hydra species that were used in this study could be categorized into the four groups by Campbell's keys. Both molecular phylogenetic analyses using the mitochondrial genes COI, $\operatorname{lr} R N A-t R N A(W)-C O I I$ and nuclear genes CnNOS1, EF1a indicated that all analyzed hydra species were clustered into four monophyletic groups (Fig. 1 - 3 and Supple. Fig. 1 - 3). This clustering of four monophyletic species groups is consistent with Campbell's suggested grouping, the viridissima group, braueri group, oligactis group and vulgaris group. Although only the M2 strain had been identified as an oligactis group species (labeled $H$. oligactis on the list of the NIG collection), our molecular phylogenetic analyses suggested that it was a member of the carnea 
sub-group in the vulgaris group. Then, we observed morphological key characters (structure of holotrichous isorhiza) of M2 strain polyps, and confirmed that M2 is a member of the vulgaris group (Fig. 5). Therefore, the presence of four monophyletic distinct species groups in the genus Hydra was affirmed based on the both morphological data and nucleotide sequence data. The molecular analysis on the eight members of the genus Hydra by Hemmrich et al. (2007) also supports the presence of four distinct species groups in hydra.

However, more than half of the strains used in this study originally had been collected in Japan. The sampling locations of the other strains were restricted to Europe and North America. Although, the genus Hydra has only a few dozens species, they have been recorded from all over the world excepting Antarctica (Jankowski, Collins and Campbell, 2008). To understand the entire phylogenetic relationship in the genus Hydra, we need a larger collection of hydra strains involving samples from Africa, Asia, Oceania, and South America.

4.2 Molecular phylogenetic relations between strains in a same group and a same sub-group in vulgaris group

In the viridissima group, North American strains (L9, M8) always made a monophyletic clade with the K10 strains originated in Switzerland as a sister group to the other all strains, M9, M10, and J8 in our analyses (Fig.1 - 3). M9 and M10 were collected in Israel and Danube, respectively. Although migration ability of hydra is low, hydra distributed in all continent without Antarctica. Therefore, it is reasonable to presume ancestor of hydra species originated before the separation of landmass, Pangea. Jankowski et al. (2008) suggested that the viridissima group and the vulgaris group were probably present before the separation of northern and southern landmasses. Before the North America and Europe were disrupted, they had made northern landmass, Laurasia. In the Laurasia, North America and West Europe were connected each other. If the ancestor of these viridissima group strains had originated in the middle part of the Laurasia (East Europe), it was probable that descendants dispersed to the west made the ancestors of L9, M8 strains and those dispersed to the east or stayed original location made the ancestors of M9, M10 strains. Recently, new green hydra, H. sinensis, was described from China (Wang et. al., 2009). It is interesting to identify the phylogenetic position of this new species in the viridissima group. 
In the oligactis group, Japanese strains (B1, B2, B3, L5, and P) formed a monophyletic clade against the other European (G7, K12) and North American (M4) strains (Fig. 1 and Supple. Fig. 1A - C). This result is consistent with Campbell's key (1983). According to Campbell's key, holotrichous isorhiza without transverse coils is also a trait of the oligactis group species excepting Hydra pseudoligactis (=Hydra canadensis). Such hydra possessing holotrichous isorhiza without transverse coils from Japan is Hydra robusta (= Pelmatohydra robusta, Ito), and from elsewhere is Hydra oligactis. As was the case of viridissima group, it was probable that ancestor of oligactis group strains originated in the middle part of Laurasia then dispersed to the west and east before the separation of the continent and diverged. Hydra robusta (B1, B2, B3, L5, and P) might be the descendant of such ancestor in the east end of the Laurasia, and the ancestor dispersed to the west might generate Hydra oligactis (G7, K12, M4).

In the braueri group, we only analyzed three species, H. hymanae (North America), H. utahensis (North America), and H. circumcincta (Europe). In our analyses, circumcincta (K8, M7) and utahensis (L10) made a monophyletic clade as a sister group to the hymanae (M1). We could not explain this inconsistency between the geographic distribution and phylogenetic relation among the braueri group species.

The genetic distance between strains within the vulgaris group was small (Fig. 1). However, three sub-groups were recognized distinctly in the vulgaris group (Fig. 1 3). The grouping of the three sub-groups, however, did not reflect the geographic distribution. Analyzed vulgaris sub-group strains all collected in Europe or Japan. In the carne sub-group strains, analyzed samples were collected from North America and Japan. Hydra sp. (K5, K6) sub-group strains were collected in Europe.

Of the vulgaris sub-group strains analyzed in this study, only four strains, B6, L2, K9, K7, were collected in Europe and all other strains (more than twenty) were collected in Japan. Although, three strains of them, B6, L2, and K9, formed a monophyletic clade, the strain K7 was clustered with other Japanese strains (Fig. 1 and Supple. Fig. 1A - C). Because only a small number of strains except for Japanese ones were analyzed in this study, we could not say much about the phylogenetic relationships in the vulgaris sub-group. However, it was suggested that members of the vulgaris sub-group were distributed in the Eurasia, but not in North America. The genetic distances between strains in the vulgaris sub-group were very small and the molecular 
phylogenetic relations among vulgaris sub-group did not reflect the geographic distribution.

One of the other sub-groups consists of only two strains, K5 and K6, which had been identified as Hydra attenuata in the NIG collection. These strains had been cloned in 1962 and added to the NIG collection from Prof. Tardent's laboratory in 1988. However, Campbell (1989) suggested that Hydra attenuata was a synonym of Hydra vulgaris. Although K5 and K6 belong to Hydra vulgaris according to Campbell's suggestion, they had some morphological traits that were distinctly different from those of Hydra vulgaris as described in the results (Fig. 4 and 5). Therefore, we suggested that $\mathrm{K} 5$ and $\mathrm{K} 6$ form an independent sub-group in the vulgaris group.

All other strains in the vulgaris group belonged to the carnea sub-group in this molecular phylogenetic analysis. Although the M5 strain (USA CA) had been identified as Hydra vulgaris in the NIG collection, it belonged to the carnea sub-group by the molecular phylogenetic analyses. The M2 strain (USA CA), which had been identified as a member of the vulgaris group based on the morphological traits in this study, also belonged to the carnea sub-group. Although, only four stains, AEP, L4, M2, and M5, were analyzed in this study, the result suggested that there is only the carnea sub-group and no other sub-group of the vulgaris group in North America. There are, of course, several hydra species within the vulgaris group that have been described from North America, such as H. littoralis, H. cauliculata, H. rutgersensis (Campbell, 1983). It is interesting to see whether these North American vulgaris group species belong to the carnea sub-group or not. The M2 and M5 strains may be one of those species, because these two strains made a sister clade in relation to all other carnea sub-group (Fig. 1 and Supple. Fig 1A - C).

Although North American vulgaris group strains all belonged to the carnea sub-group, the distribution of the carnea sub-group was not restricted in the North America. Newly collected strains, FT 08, FO2 03, KAS 20, in Japan also belonged to the carnea sub-group.

\subsection{Japanese vulgaris group hydra}

Five species of hydra have been described from Japan (Ito, 1947a, b, c, d). Three of them, H. magnipapillata, H. japonica, and $H$. paludicola, belong to the vulgaris group, and maybe the same species. The standard wild type strain, Hydra 
magnipapillata 105 (=B8 strain in this study), has been used in many researches. Recently, draft assemblies of the Hydra magnipapillata genome were generated (Chapman et al., 2010). Therefore, we thought it appropriate that there is only one hydra species belonging to the vulgaris sub-group in Japan, and it is Hydra magnipapillata.

However, in the course of our phylogenetic analyses, we found that newly collected hydra species from three different locations (Kasuga City, Tanushimaru in Kurume City and Ohori in Fukuoka City) in Fukuoka prefecture, Japan, belonged to the carnea sub-group (Fig. 1, FT 08, FO2 03, KAS 20). In the Japanese strains of the NIG collection, however, no strains belonged to the carnea sub-group, and all of them belonged to the vulgaris sub-group. We, of course, also collected vulgaris sub-group $(H$. magnipapillata) strains in Fukuoka, FO1 G, FO2 05, and ONI 5.Therefore, our molecular phylogenetic analyses suggested there were at least two species belonging to the vulgaris group in Japan, Hydra magnipapillata and Hydra sp. that belonged to the carnea sub-group. Members of the vulgaris sub-group are distributed in Eurasia but not in North America while those in the carnea sub-group are distributed in North America but not in Europe. Campbell (1983) described as well known vulgaris group species Hydra littoralis (North America), Hydra attenuata (= Hydra vulgaris) (Europe), and Hydra magnipapillata (Japan). Therefore, it is very interesting that both the vulgaris and the carnea sub-groups distribute in Japan.

However, most of the carnea sub-group samples in Japan were collected in urban area in the Fukuoka prefecture except the FT 08 strain. Therefore, we could not exclude the ability of anthropogenic dispersal from USA. However, we also could not exclude the ability a trans-Pacific distribution of the carnea sub-group hydra. We need information on hydra strains that inhabit Siberia and main land China for considering this interesting distribution of hydra species.

\subsection{Hydra population in a pond}

We found that, in all ponds from which multiple polyps were collected in our study, all polyps belonging to the vulgaris sub-group from the same pond had the same MtDNA nucleotide sequence. We cold not find any substitution in analyzed nucleotide sequences. The usual hydra reproduction system, asexual budding, provides a plausible explanation for this result. Interestingly, however, a vulgaris sub-group strain, FO2 05 (this sample represented three samples), and a carnea sub-group strain FO2 03 (this 
sample also represented three samples) were collected in a same pond. The diameter of this small pond was about $20 \mathrm{~m}$. We only analyzed the mitochondrial nucleotide sequence data. Therefore, we could not certain whether hybridization between FO2 05 (vulgaris sub-group) and FO2 03 (carnea sub-group) has occurred or not. To certain this problem is very intriguing.

\subsection{Insertion in the mitochondrial DNA in baurei group}

In the four species groups of hydra, insertions in mitochondrial DNA were observed only in the braueri group species. In the three other groups, $\operatorname{lr} R N A, \operatorname{tRNA}(W)$ and COII coding region did not contain any insertions. This is a remarkable feature of the MtDNA evolution in the braueri group species.

In H. utahensis (L10) the inserted DNA suggested to have been originated from the $t R N A-C O I I$ coding region based on the nucleotide sequence data (Supple. Fig. 4). The inserted location was between the $\operatorname{lr} R N A$ region and the $t R N A(W)$ region. Duplication of this region and following modification was suggested. However, the inserted DNA sequence in H. hymanae (M1) did not any homology or similarity with any known DNA sequences. Therefore, it was suggested that origins of these insertions were different and the insertions had not occur in the common ancestor of the both strains. However, the MtDNA insertion of H. hymanae (M1) and of H. utahensis occurred in vicinity. The $\operatorname{lr} R N A$ - $t R N A(W)$ - COII region in braueri group hydra may have a specific character of nucleotide sequence to induce insertions.

4.5 Substitution rate of nucleotide sequence is higher in mitochondrial genes than that of nuclear genes in hydra species

The difference of substitution rate between mitochondrial genome and nuclear genome has been an intriguing problem in the molecular evolutionary researches. The differences of substitution rate have been reported in several animals. Hydra polyps reproduce mainly asexually by budding, and have linear, not circular, mitochondrial genome. Therefore, it has been intriguing to investigate differences of the substitution rate between the mitochondrial genome and nuclear genome in hydra species.

Higher synonymous substitution rates in mitochondrial genes than in nuclear genes have been reported in animals. For example, it was reported that the rate of MtDNA evolution in primates is 5 to 10 times higher than in nuclear DNA (Brown et al., 
1979, 1982). Moriyama and Powell (1997) reported that mitochondrial genes have 1.73.4 times higher synonymous substitution rates than the fastest nuclear genes or 4.5-9.0 times higher rates than the average nuclear genes in Drosophila. On the other hand, lower Metazoa, such as Polrifera and Anthozoa, have been shown to have a reduced mitochondrial DNA substitution rate compared to nuclear DNA. In coral, 3 to 10 times slower rates of MtDNA sequence substitution compared with nuclear DNA were reported (reviewed by Shearer et al., 2002). Hellberg (2006) suggested that Anthozoa in Cnidaria showed slow synonymous substitution rate of MtDNA. However, Medusozoa, including Hydrozoa, showed faster rate as is the case of Bilateria.

Under a good condition with appropriate temperature and rich preys, hydra species reproduce rapidly by asexually budding. However, hydra species show sexual reproduction depending on the environmental condition. In the sexual reproduction phase, hydra species show diverse styles. Both monoecious species (the viridissima group, the braueri group, and some in the vulgaris group) and dioecious species (the oligactis group and most cases in the vulgaris group) with sexual differentiation have been reported. Because asexual reproduction is expected to decrease mutation rates at nuclear genes to retard accumulation of deleterious mutations (Kondrashov, 1988), it is interesting to know whether the ratio of synonymous rates in mitochondrial and nuclear genes differs from those in sexual species. Our result showed that this ratio in hydras was 2.4-7.8 and comparable to those in primates (Brown et al., 1979, 1982) and Drosophila (Moriyama and Powell, 1997). Therefore, partial asexual reproduction does not seem to reduce the nuclear mutation rate or some other factors such as lower mutation rates in MtDNA that have been observed in Anthozoa might have erased this effect.

In the estimation of time of speciation and phylogeographic events, knowing neutral evolutionary rates is a prerequisite. Furthermore, many questions in population genetics depend on information of mutation rates that can be estimated from neutral evolutionary rates (Kimura 1983). Finally, one of the main reasons why mitochondrial CO1 was chosen for DNA barcoding in many animals is the higher evolutionary rate of mitochondrial genes in those species (Herbert et al., 2003). Therefore, it is important to obtain information of evolutionary rates when evolutionary questions are to be asked in hydras. Of course, we obtained only the ratio of the evolutionary rates in MtDNA and nuclear DNA, and not their absolute rates. This situation needs to be rectified in future 
studies although we expect difficulties in calibrating time in organisms with soft bodies like hydras. Nevertheless, the higher rate in MtDNA compared to that in the nuclear genome in hydra as found in other animals indicate usefulness of MtDNA genes as markers for evolutionary studies of short time scales.

\section{Conclusion}

Species in the genus Hydra could be grouped into four monophyletic species groups by the molecular phylogenetic analyses in this study. The four hydra species groups were concordant with Campbell's suggested four groups, the viridissima group, braueri group, oligactis group and vulgaris group. Distinguishing between these four groups is easy by Campbell's keys based on the morphological traits. However, within each group it seems too difficult to distinguish between species only by the morphological key traits. As shown in this study, molecular data have an advantage in the identification of hydra species and phylogenetic analyses of the genus Hydra. However, we could only use several dozens samples from Europe, North America and Japan in this study, so in order to know the entire phylogenetic relationships in the genus Hydra, we need to conduct molecular phylogenetic studies on larger collections of hydra strains involving samples from Africa, Asia, Oceania, and South America.

\section{Acknowledgments}

We would like to thank two reviews for their pointed comments and suggestions. They were very helpful to revise our manuscript. 


\section{References}

Bosch, T.C.G., Fujisawa, T., 2001. Polyps, peptides and patterning. BioEssays 23 (5), $420-427$.

Bosch, T.C.G., Khalturin, K., 2002. Patterning and cell differentiation in Hydra: novel genes and the limits to conservation. Can. J. Zoolog. 80,1670-1677.

Bosch, T.C.G., 2009. Hydra and the evolution of stem cells. BioEssays 31, 478-486.

Bosch, T.C.G., Anton-Erxleben, F., Hemmrich, G., Khalturin, K., 2010. The Hydra polyp: Nothing but an active stem cell community. Dev. Growth Differ. 52, $15-25$.

Bridge, D., Cunningham, C.W., Schierwater, B., DeSalle, R., Buss, L.W., 1992. Class-level relationships in the phylum Cnidaria: Evidence from mitochondrial genome structure. Proc. Natl. Acad. Sci. USA, 89, 8750-8753.

Bridge, D., Cunningham, C.W., DeSalle, R., Buss, L.W., 1995. Class-level relationships in the phylum Cnidaria: molecular and morphological evidence. Mol. Biol. Evol. 12 (4), 679-689.

Brown, W. M., George, M. Jr., Wilson, A. C., 1979. Rapid evolution of animal mitochondrial DNA. PNAS. 76(4). 1967-1971.

Brown, W. M., Prager, E. M., Wang, A., Wilson, A. C., 1982. Mitochondrial. DNA sequences of primates: Tempo and mode of evolution. J. Mol. Evol. 18. 225-239.

Campbell, R.D., 1983. Identifying Hydra species. In: Lenhov, H.M. (Ed.), Hydra: Research Methods. Plenum Press, New York and London, pp. 9-28. Chapter 3. 
Campbell, R.D., 1987. A new species of Hydra (Cnidaria: Hydrozoa) from North America with comments on species clusters within the genus. Zool. J. Linn. Soc-Lond. 91, 253-263.

Campbell, R.D., 1989. Taxonomy of the European Hydra (Cnidaria: Hydrozoa): a re-examination of its history with emphasis on the species $\mathrm{H}$. vulgaris Pallas, $\mathrm{H}$. attenuata Pallas and H. circumcincta Schulze. Zool. J. Linn. Soc-Lond. 95 (3), 219-244.

Chapman, J.A. et al., 2010. The dynamic genome of Hydra. Nature, 464, 592-596.

Collins, A.G., 2000. Towards understanding the phylogenetic history of Hydrozoa: hypothesis testing with $18 \mathrm{~S}$ gene sequence data. Sci. Mar., 64, 5-22.

Collins, A.G., 2002. Phylogeny of the Medusozoa and the evolution of cnidarian life cycles. J. Evol. Biol. 15, 418-432.

Collins, A.G., Schuchert, P., Marques, A.C., Jankowski, T., Medina, M., Schierwater, B., 2006. Medusozoan phylogeny and character evolution clarified by new large and small subunit rDNA data and an assessment of the utility of phylogenetic mixture models. Syst. Biol. 55, 97-115.

Dumont, H.G., 1994. The distribution and ecology of the fresh- and brackish-water medusae of the world. Hydrobiologia 272, 1-12.

Felsenstein, J., (1985). Confidence limits on phylogenies: An approach using the bootstrap. Evolution 39, 783-791.

Galtier, N., Gouy, M., Gautier, C., 1996. SeaView and Phylo_win, two graphic tools for sequence alignment and molecular phylogeny. Comput. Applic. Biosci. 12, 543-548.

Guindon, S., Gascuel, O., 2003. A simple, fast, and accurate algorithm to estimate large phylogenies by maximum likelihood. Systematic Biology 52, 696-704. 
Hebert, P.D.N., Cywinska, A, Ball, S.L., deWaard, JR, (2003) Biological identifications through DNA barcodes. Proc. R. Soc. Biol. Sci. Ser. B 270, 313-321.

Hellberg, M.E., 2006. No variation and low synonymous substitution rates in coral mtDNA despite high nuclear variation. BMC Evolutionary Biology, 6, 24 doi:10.1186/1471-2148-6-24

Hemmrich, G., Anokhin, B., Zacharias, H., Bosch, T.C.G., 2007. Molecular phylogenetics in Hydra, a classical model in evolutionary developmental biology. Mol Phylogenet Evol. 44, 281-90.

Holstein, T.W., Hobmayer, E., Technau, U., 2003. Cnidarians: an evolutionarily conserved model system for regeneration? Dev. Dyn. 226, 257-267.

Huelsenbeck, J.P., Ronquist, F., 2001. MRBAYES: bayesian inference of phylogeny. Bioinformatics 17, 754-755.

Ito, T., 1947. On a new species of fresh-water polyp from Japan. Science reports of The Tohoku Univ. 4th Ser. (Biology) 18, 1-5.

Ito, T., 1947. A new fresh-water polyp, Hydra magnipapillata, N. SP. from Japan. Science reports of The Tohoku Univ. 4th Ser. (Biology) 18, 6-10..

Ito, T., 1947. Description of a new Pelmatohydra from Japan. Science reports of The Tohoku Univ. 4th Ser. (Biology) 18, 11-16.

Ito, T., 1947. Two new species of fresh-water polyp from Japan. Science reports of The Tohoku Univ. 4th Ser. (Biology) 18, 17-23.

Jankowski, T., Collins, A.E., Campbell, R., 2008. Global diversity of inland water cnidarians. Hydrobiologia 595, 35-40. 
Kayal, E., Lavrov, D.V., 2008. The mitochondrial genome of Hydra oligactis (Cnidaria, Hydrozoa) sheds new light on animal mtDNA evolution and cnidarian phylogeny. Gene 410, 177-186.

Kimura, M., 1983. The Neutral Theory of Molecular Evolution. Cambridge University Press, Cambridge.

Kondrashov, A. S., 1988. Deleterious mutations and the evolution of sexual reproduction. Nature 336: 435-440

Kumar. S., Dudley, J., Nei, M., Tamura, K., 2008. MEGA: A biologist-centric software for evolutionary analysis of DNA and protein sequences. Briefings in Bioinformatics 9: 299-306.

Moriyama, E. N., Powell, J. R., 1997. Synonymous substitution rates in Drosophila: mitochondrial versus nuclear genes. J. Mol. Evol. 45(4). 378-391

Nei, M., Gojobori, T., 1986. Simple methods for estimating the numbers of synonymous and nonsynonymous nucleotide substitutions. Molecular Biology and Evolution 3, 418-426.

Nei, M., Kumar, S., (2000) Molecular Evolution and Phylogenetics. Oxford University Press, New York.

Nielsen, C., 2001. Phylum Cnidaria. In: Animal evolution $2^{\text {nd }}$ ed. Oxford University Press Inc, New York, pp. 59-69. Chapter 8.

Posada, D. 2008. jModelTest: Phylogenetic Model Averaging. Mol. Biol. Evol. 25, 1253-1256.

Saitou, N., Nei, M., 1987. The neighbor-joining method: A new method for reconstructing phylogenetic trees. Molecular Biology and Evolution 4, 406-425. 
Shearer, T. L., van Oppen, M. J. H., Romano, S. L., Wörheide, G., 2002. Slow mitochondrial DNA sequence evolution in the Anthozoa (Cnidaria). Molecular Ecology, 11, 2475-2487.

Steele, R.E., 2002. Developmental signaling in Hydra: what does it take to build a "simple" animal? Dev. Biol. 248 (2), 199-219.

Tamura, K., Dudley, J., Nei, M., Kumar, S., 2007. MEGA4: Molecular Evolutionary Genetics Analysis (MEGA) software version 4.0. Molecular Biology and Evolution 24: $1596-1599$.

Thomsen, S., Bosch T.C.G., 2006. Foot differentiation and genomic plasticity in Hydra: lessons from the PPOD gene family. Dev Genes Evol, 216, 57-68.

Thompson, J.D., Higgins, D.G., Gibson, T.J., 1994. CLUSTAL W: improving the sensitivity of progressive multiple sequence alignment through sequence weighting, positions-specific gap penalties and weight matrix choice. Nucleic Acids Research, 22:4673-4680.

Thompson, J.D., Gibson,T.J., Plewniak, F., Jeanmougin, F., Higgins, D.G., 1997. The ClustalX windows interface: flexible strategies for multiple sequence alignment aided by quality analysis tools. Nucleic Acids Research, 24:4876-4882.

Tudge C., 2000. The variety of Life. Oxford University Press Inc, New York, pp. 211-224. Chapter 6.

Voigt, O., Erpenbeck, D., Wörheide, G., 2008. A fragmented metazoan organellar genome: the two mitochondrial chromosomes of Hydra magnipapillata. BMC Genomics, 9:350

Wang An-Tai, Li Deng, Jing-Qi Lai and Juan Li. (2009) A New Species of Green Hydra (Hydrozoa: Hydrida) from China. Zool. Sci., 26, 664-668. 
Wittlieb, J., Khalturin, K., Lohmann, J., Anton-Erxleben, F., Bosch, T. C. G., 2006. Transgenic Hydra allow in vivo tracking of individual stem cells during morphogenesis. Proc. Natl. Acad. Sci. USA 103, 6208-6211. 


\section{Figure captions}

\section{Figure 1:}

Molecular phylogenetic tree (unrooted) based on maximum likelihood phylogenetic analysis of the combined mitochondrial dataset, containing the COI gene and IrRNA-tRNA (W)-COII genes. Numerals near the nodes indicate bootstrap values (\%) based on 1000 replicates. Fifty-five strains of hydra were analyzed. The branch length indicator displays 0.05 substitutions per site. Four main clades, the viridissima, braueri, oligactis and vulgaris groups were recognized and indicated by the rectangular field with solid lines. Rectangular fields with dotted line indicate three sub-clades in the vulgaris group, vulgaris sub-group, carnea sub-group, and Hydra sp. (K5, K6) sub-group. These clades were also recognized by the NJ, MP, and BI analyses (See Supple. Fig. 1A, B, C). On the nodes of those clades, bootstrap values of the NJ (10000), MP (1000) analyses and posterior probabilities of the BI analysis are also indicated (\%) with bold face (order; ML/NJ/MP/ BI).

\section{Figure 2:}

Molecular phylogenetic tree (unrooted) based on the maximum likelihood (ML) phylogenetic analysis of the CnNOS1 gene. Numerals near the nodes indicate bootstrap values (\%) based on 1000 replicates. Twenty-five strains of hydra were analyzed. The branch length indicator displays 0.02 substitutions per site. Four major clades were also recognized as in the case with the mitochondrial gene analyses (Fig. 1). On the nodes of major clades, which were recognized in NJ, MP, and BI analyses (See Supple. Fig. 2A, B, C), bootstrap values of the ML (1000), NJ (10000), MP (1000), analyses and posterior probabilities of the BI analysis (order; ML/NJ/MP/BI) were also indicated (\%) with bold face.

\section{Figure 3:}

The molecular phylogenetic tree (unrooted) based on the maximum likelihood (ML) phylogenetic analysis of the EFla gene. Twenty-five strains of hydra were analyzed. Numerals near the nodes indicate bootstrap values (\%) based on 1000 replicates. The branch length indicator displays 0.01 substitutions per site. Four main clades were also recognized as in the case with the mitochondrial gene analyses (Fig.1). 
On the nodes of major clades, which were recognized in NJ, MP, BI analyses (See

Supple. Fig. 3A, B, C), bootstrap values of the ML (1000), NJ (10000), MP (1000), analyses and posterior probabilities of the BI analysis were indicated (\%) with bold face (order; ML/NJ/MP/BI).

\section{Figure 4:}

A: Photograph of the strain K5. Length of tentacles is shorter than that of body column. Sexual polyps, male and female were also shown. B: Magnified testes. C: Magnified fertilized egg with embryonic theca.

\section{Figure 5:}

Four types of nematocysts in 8 strains of hydra species. Each strain shows distinctive shape of nematocysts. However, it is difficult to distinguish between Hydra carnea (L4) and Hydra magnipapillata (B8, 105). 


\section{Supplemental Figure 1:}

A: Molecular phylogenetic tree based on the neighbor joining (NJ) phylogenetic analysis of the combined mitochondrial dataset, containing the $C O I$ gene locus and IrRNA-tRNA (W)-COII genes locus. The bootstrap consensus tree inferred from 10000 replicates is taken to represent the evolutionary history of the taxa analyzed. Numerals near the nodes indicate bootstrap values (\%). Four main clades, the viridissima, braueri, oligactis and vulgaris groups were recognized and indicated by the rectangular field with solid lines. Rectangular fields with dotted line indicate three sub-clades in the vulgaris group, vulgaris sub-group, carnea sub-group, and Hydra sp. (K5, K6) sub-group. The ML, MP, and BI analyses also indicated these clades. On the nodes of those clades, bootstrap values of the NJ (10000), MP (1000), ML (1000) analyses and posterior probabilities of the $\mathrm{BI}$ analysis (order; $\mathrm{NJ} / \mathrm{MP} / \mathrm{ML} / \mathrm{BI}$ ) are indicated (\%) with bold face. The branch length indicator displays 0.05 substitutions per site.

B: Maximum parsimony (MP) phylogenetic analysis of the combined mitochondrial dataset, containing COI gene and lrRNA-tRNA (W)-COII genes. Numerals near the nodes indicate bootstrap values (\%) based on 1000 replicates. On the nodes of major clades, bootstrap values of the MP (1000), NJ (10000), ML (1000) analyses and posterior probabilities of the BI analysis (order; MP/NJ/ML/BI) are indicated (\%) with bold face.

C: Bayesian inference (BI) analysis of the combined mitochondrial dataset, containing the $\mathrm{COI}$ gene and $\mathrm{COII}$ genes. Numerals near the nodes indicate posterior probabilities (\%). On the nodes of major clades, posterior probabilities of the BI analysis and bootstrap values of the NJ (10000), MP (1000), ML (1000) analyses are indicated (\%) with bold face (order; BI/NJ/MP/ML).

\section{Supplemental Figure 2:}

A: Molecular phylogenetic tree based on the neighbor joining $(\mathrm{NJ})$ phylogenetic analysis of the CnNOS1 gene. The bootstrap consensus tree inferred from 10000 replicates is shown. The evolutionary distances were computed using the Nei-Gojobori method and are in the units of the number of synonymous differences per synonymous site. Four major clades were also recognized as in the case with the mitochondrial gene analyses (Fig. 1). On the nodes of major clades, which were recognized in both MP, ML, and BI analyses, bootstrap values of the NJ (10000), MP (1000), ML (1000) analyses 
and posterior probabilities of the BI analysis were indicated (\%) with bold face (order; $\mathrm{NJ} / \mathrm{MP} / \mathrm{ML} / \mathrm{BI}$ ). The branch length indicator displays 0.05 substitutions per site.

B: Maximum parsimony (MP) phylogenetic analysis of the CnNOS1 gene. Numerals near the nodes indicate bootstrap values $(\%)$. On the nodes of major clades, which were recognized in both NJ, ML, and BI analyses, bootstrap values of the MP (1000), NJ (10000), ML (1000) analyses and posterior probabilities of the BI analysis (\%)were indicated with bold face (order; MP/NJ/ML/BI).

C: Bayesian inference (BI) analyses of the CnNOS1 gene. Numerals near the nodes indicate posterior probabilities (\%). On the nodes of major clades, which were recognized in NJ, MP, and ML analyses, posterior probabilities of the BI analysis and bootstrap values of the NJ (10000), MP (1000), ML (1000) analyses (\%)were indicated with bold face (order; BI/NJ/MP/ML).

\section{Supplemental Figure 3:}

A: Molecular phylogenetic tree based on the neighbor joining (NJ) analysis of the EFIa gene. The bootstrap consensus tree inferred from 10000 replicates is shown. The evolutionary distances were computed using the Nei-Gojobori method and are in the units of the number of synonymous differences per synonymous site. Four main clades were also recognized as in the case with the mitochondrial gene analyses (Fig.1). On the nodes of major clades, which were recognized in both ML, MP, BI analyses, bootstrap values (\%) of the NJ (10000), MP (1000), ML (1000) analyses and posterior probabilities of the BI analysis were indicated (\%) with bold face (order; NJ/MP/ML/BI). The branch length indicator displays 0.05 substitutions per site.

B: Maximum parsimony (MP) phylogenetic analysis of the EFla gene. Numerals near the nodes indicate bootstrap values (\%). On the nodes of major clades, which were recognized in NJ, ML, BI analyses, bootstrap values (\%) of the MP (1000), NJ (10000), ML (1000) analyses and posterior probabilities of the BI analysis were indicated (\%) with bold face (order; MP/NJ/ML/BI).

C: Bayesian inference (BI) analysis of the EFla gene. Numerals near the nodes indicate posterior probabilities (\%). On the nodes of major clades, which were recognized in NJ, MP, ML analyses, posterior probabilities of the BI analysis and bootstrap values of the NJ (10000), MP (1000), ML (1000) were indicated (\%) with bold face (order; $\mathrm{BI} / \mathrm{MP} / \mathrm{NJ} / \mathrm{ML})$. 


\section{Supplemental Figure 4:}

Dot matrix between predicted amino acid sequence of an ORF (1200bp) in the $H$. utahensis (L10) MtDNA insertion and the amino acid sequence of the COII. 
Tabale 1

Comparison between substitution rate of mitochondrial gene and of nuclear gene in Hydra

\begin{tabular}{|c|c|c|c|c|c|c|c|c|c|c|c|c|}
\hline \multirow[b]{2}{*}{ Compared strains pair } & \multicolumn{5}{|c|}{ viridissima group } & \multirow{2}{*}{$\begin{array}{l}\text { braueri } \\
\text { group } \\
\text { K8-L10 }\end{array}$} & \multicolumn{3}{|c|}{ oligactis group } & \multicolumn{3}{|c|}{ vulgaris group } \\
\hline & M9-L9 & J8-L9 & J8-K10 & M9-K10 & $\overline{L 9-K 10}$ & & L5-B3 & L5-G7 & B3-G7 & B8-J1 & B8-L4 & J1-L4 \\
\hline $\begin{array}{l}\text { Synonimoous substitution } \\
\text { rate of mitochondrial DNA } \\
(\mathrm{COI} \text { and } \mathrm{COII})\end{array}$ & 0.517 & 0.530 & 0.590 & 0.579 & 0.455 & 0.228 & 0.010 & 0.148 & 0.133 & 0.024 & 0.305 & 0.315 \\
\hline $\begin{array}{l}\text { Synonimoous substitution } \\
\text { rate of nuclear DNA } \\
(C n N O S 1 \text { and } E F 1 a)\end{array}$ & 0.208 & 0.208 & 0.184 & 0.184 & 0.102 & 0.069 & 0.040 & 0.020 & 0.017 & 0.010 & 0.051 & 0.051 \\
\hline $\begin{array}{l}\text { Synonimoous substitution } \\
\text { rate of mitochondrial DNA } \\
(\mathrm{COI} \text { and } \mathrm{COII}) / \\
\text { Synonimoous substitution }\end{array}$ & 2.486 & 2.543 & 3.207 & 3.147 & 4.461 & 3.304 & 4.000 & 7.400 & 7.824 & 2.400 & 5.000 & 6.176 \\
\hline & & & & & & & & & & & averge & 4.329 \\
\hline
\end{tabular}

CnNOS1 +Ef1a, Model : Codon: Modified Nei-Gojobori (Jukes-Cantor), Transition/Transversion Ratio : 2, Substitutions to Include : s: Synonymous only, No. of Sites : 367 COI +COII, Model : Codon: Modified Nei-Gojobori (Jukes-Cantor), Transition/Transversion Ratio : 2, Substitutions to Include : s: Synonymous only, No. of Sites : 353 
Fig.1

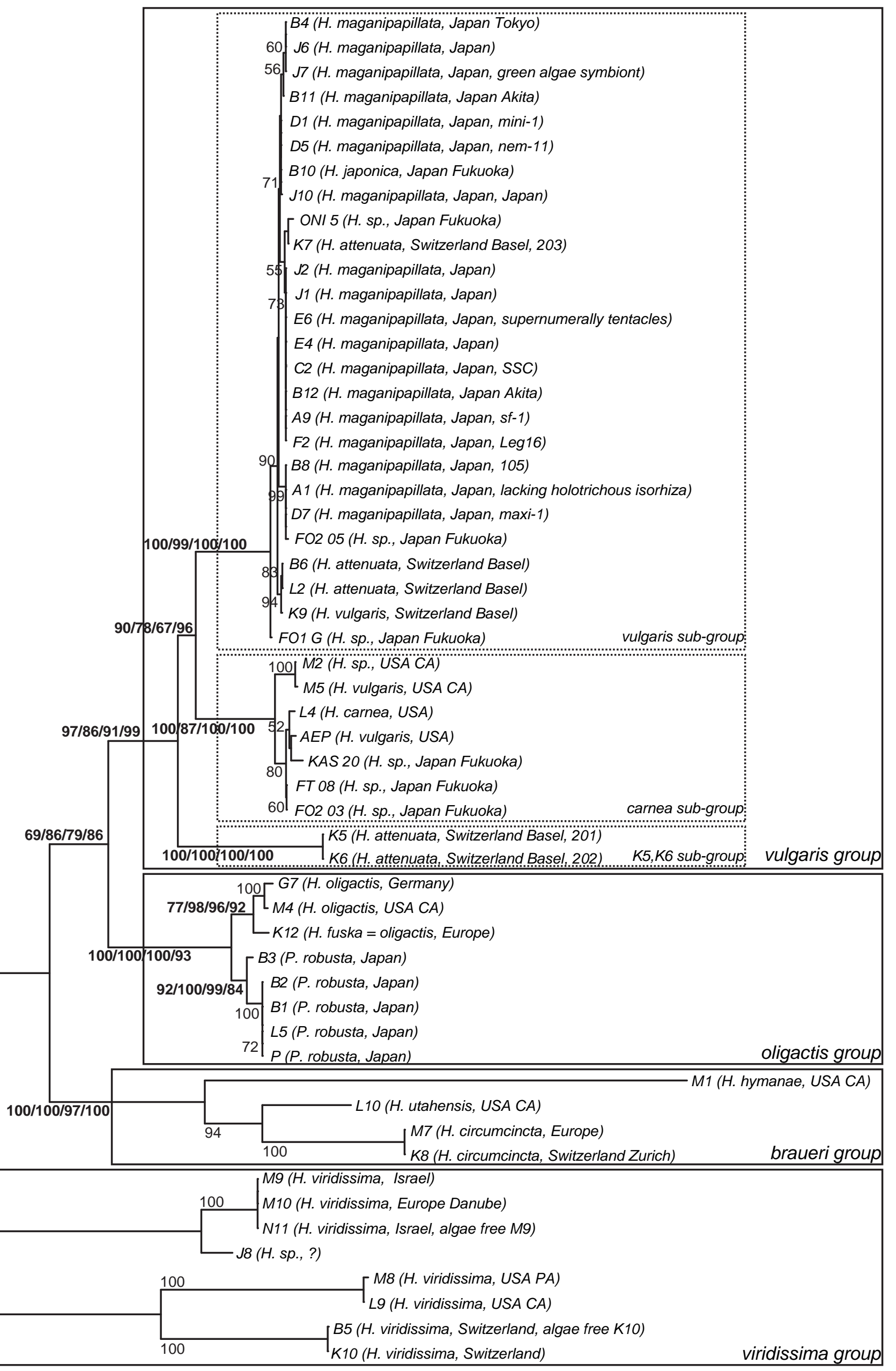




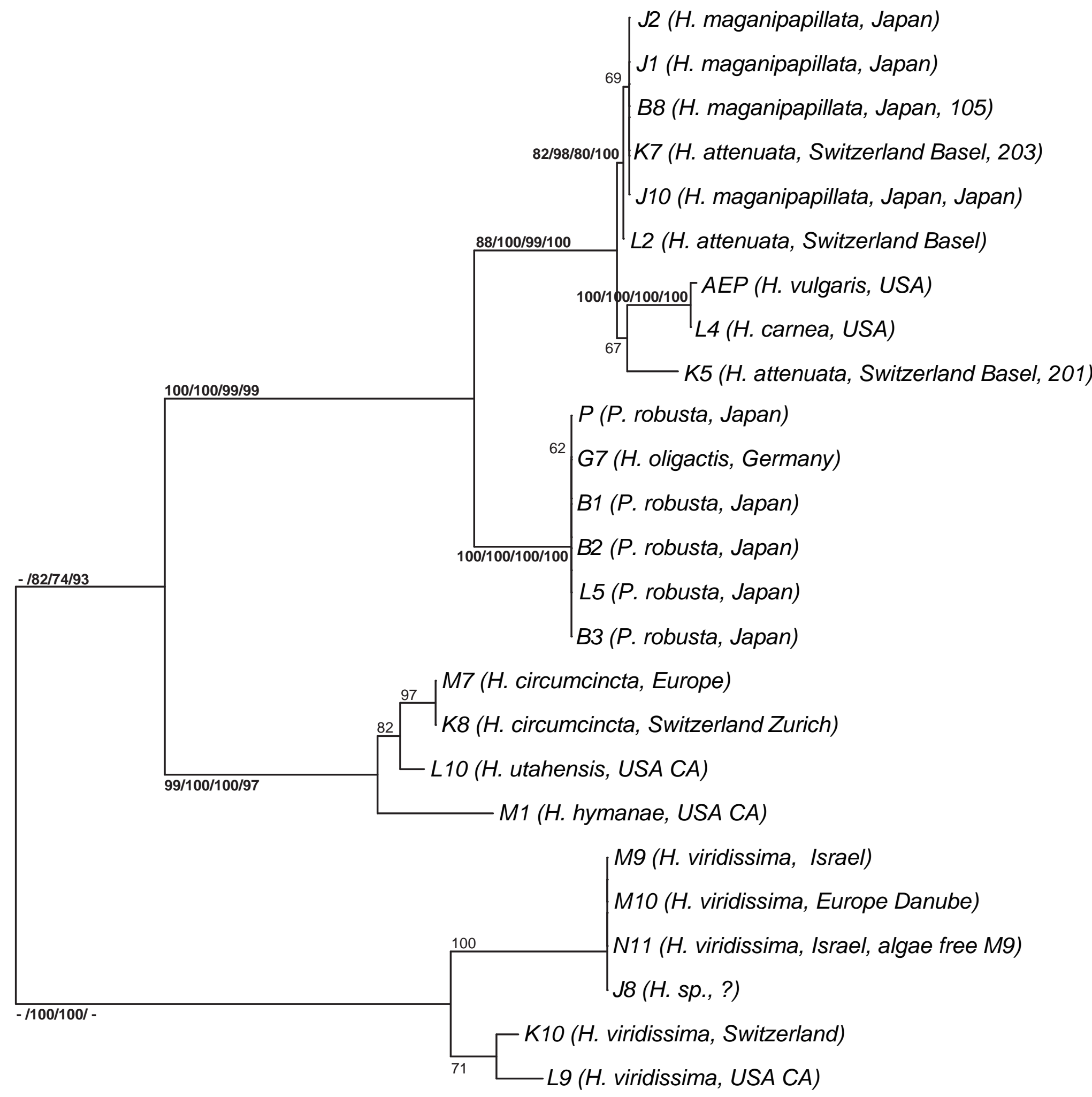


Fig.3

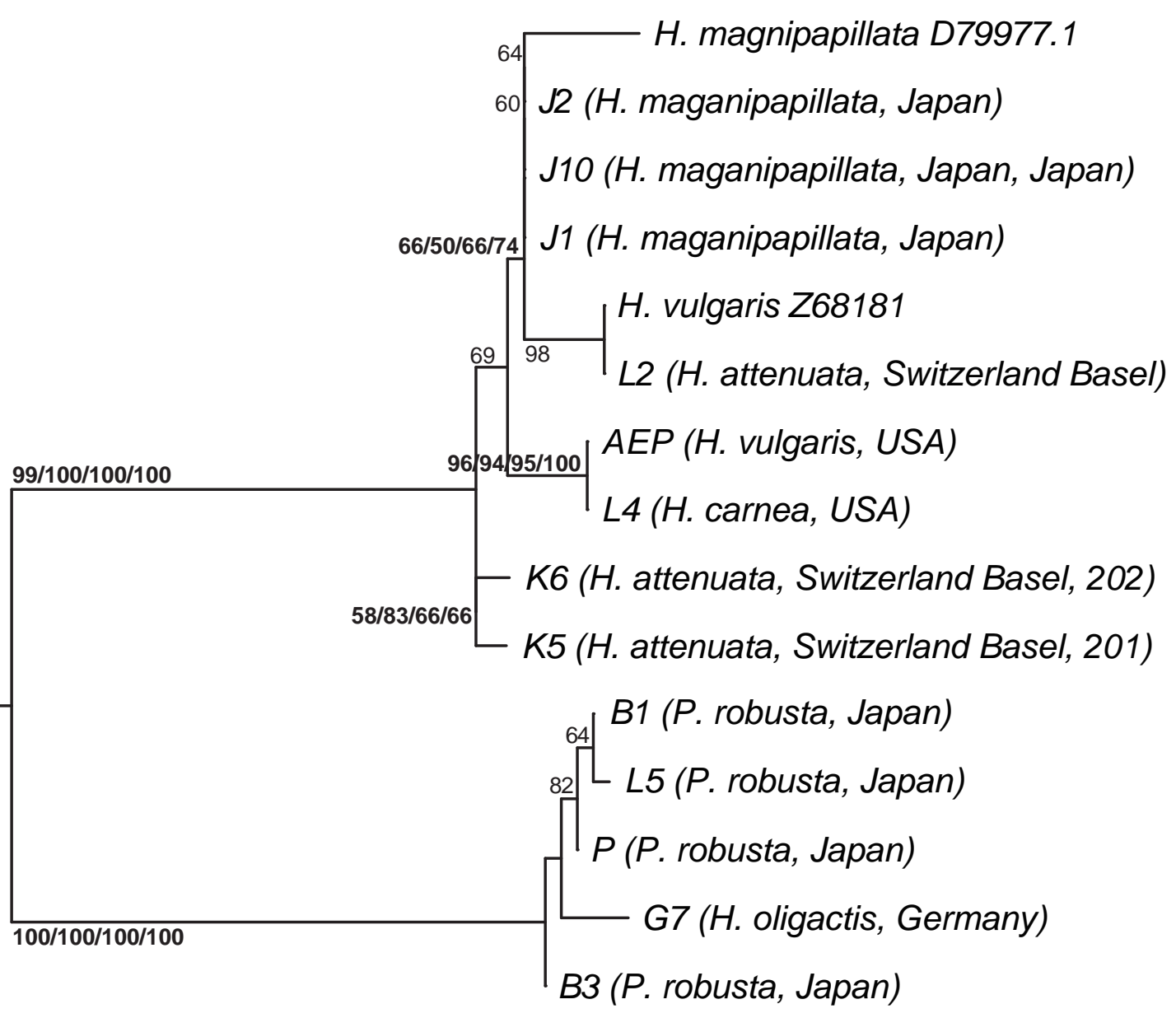


Fig.4

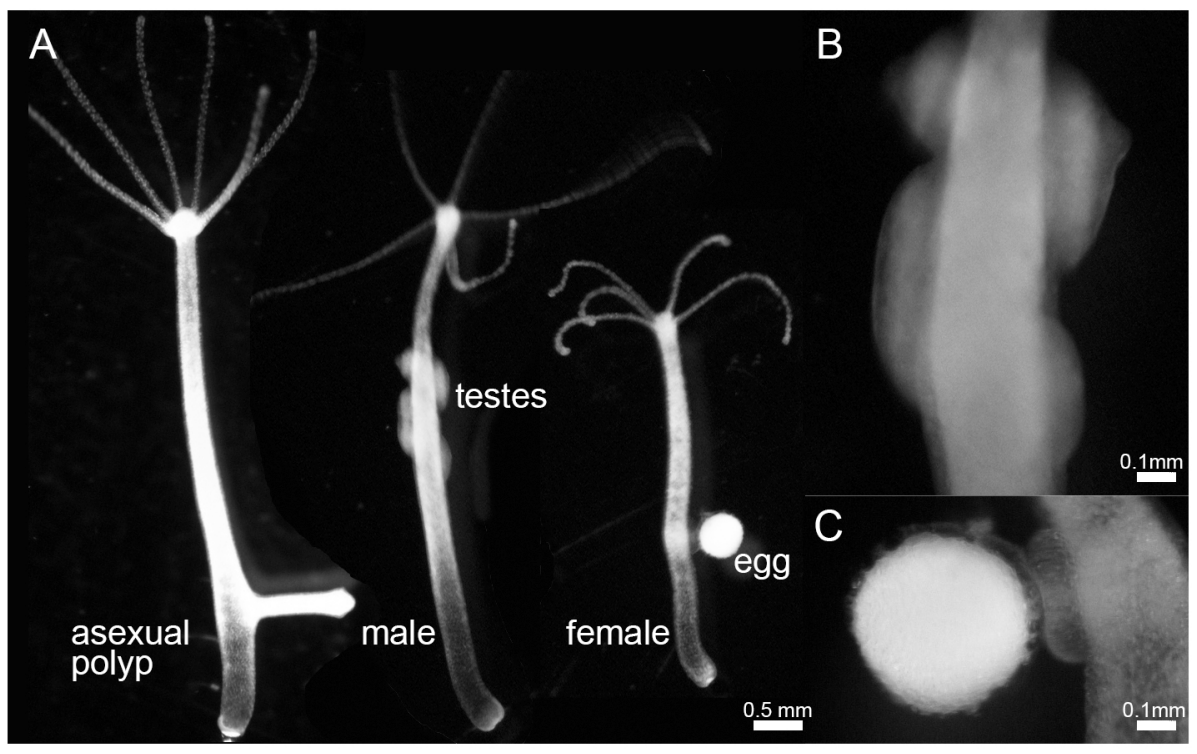


Fig. 5

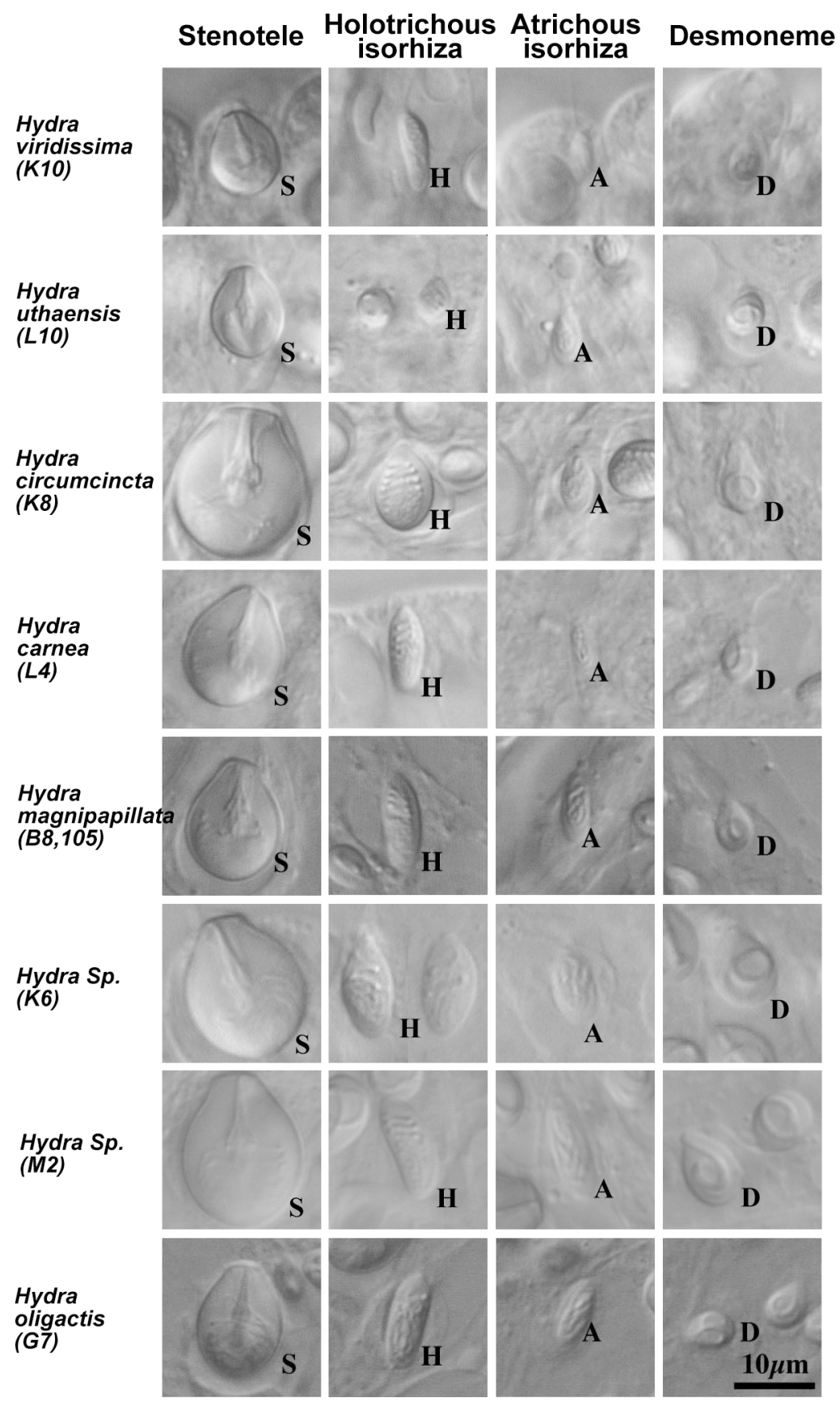




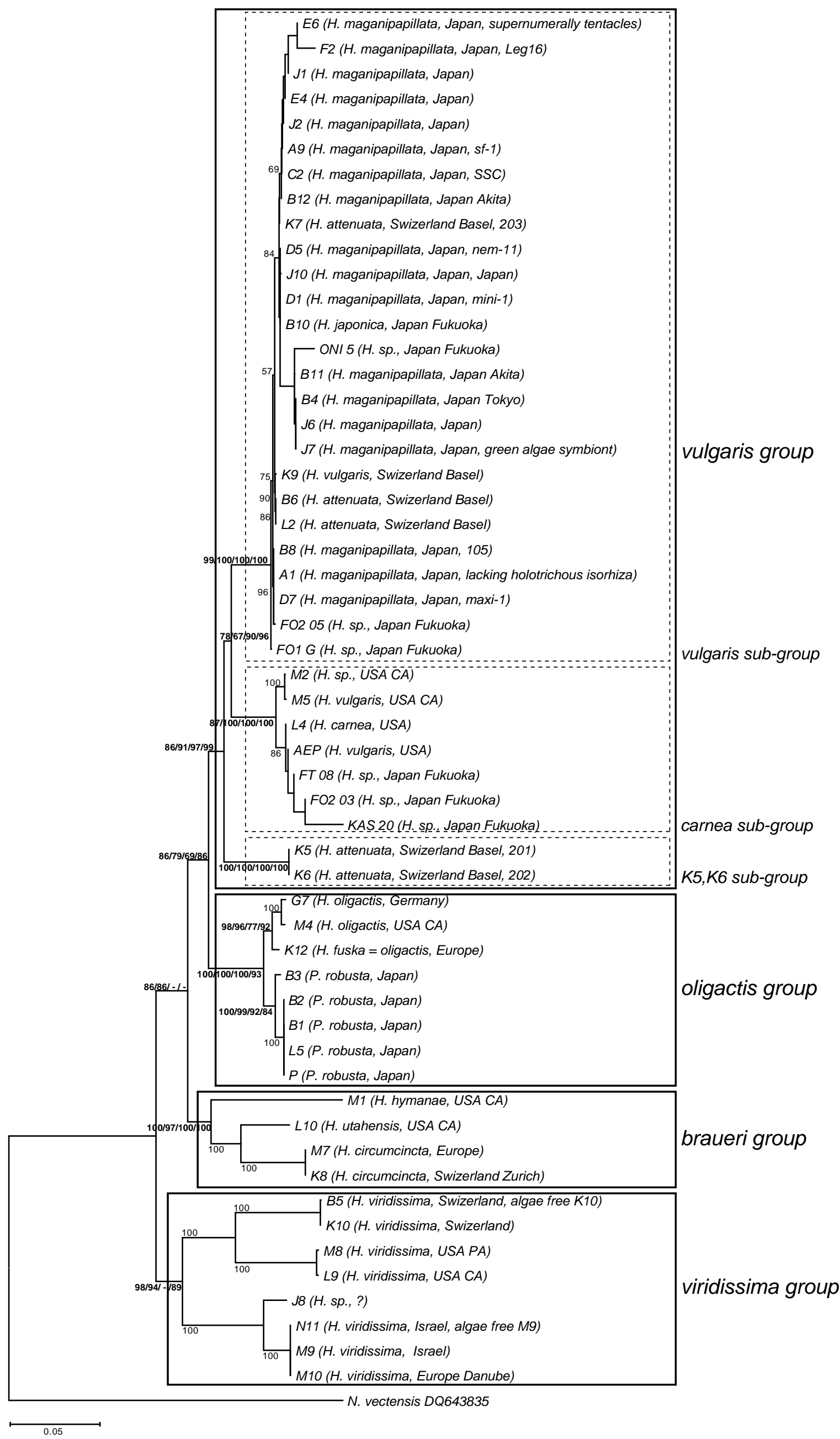




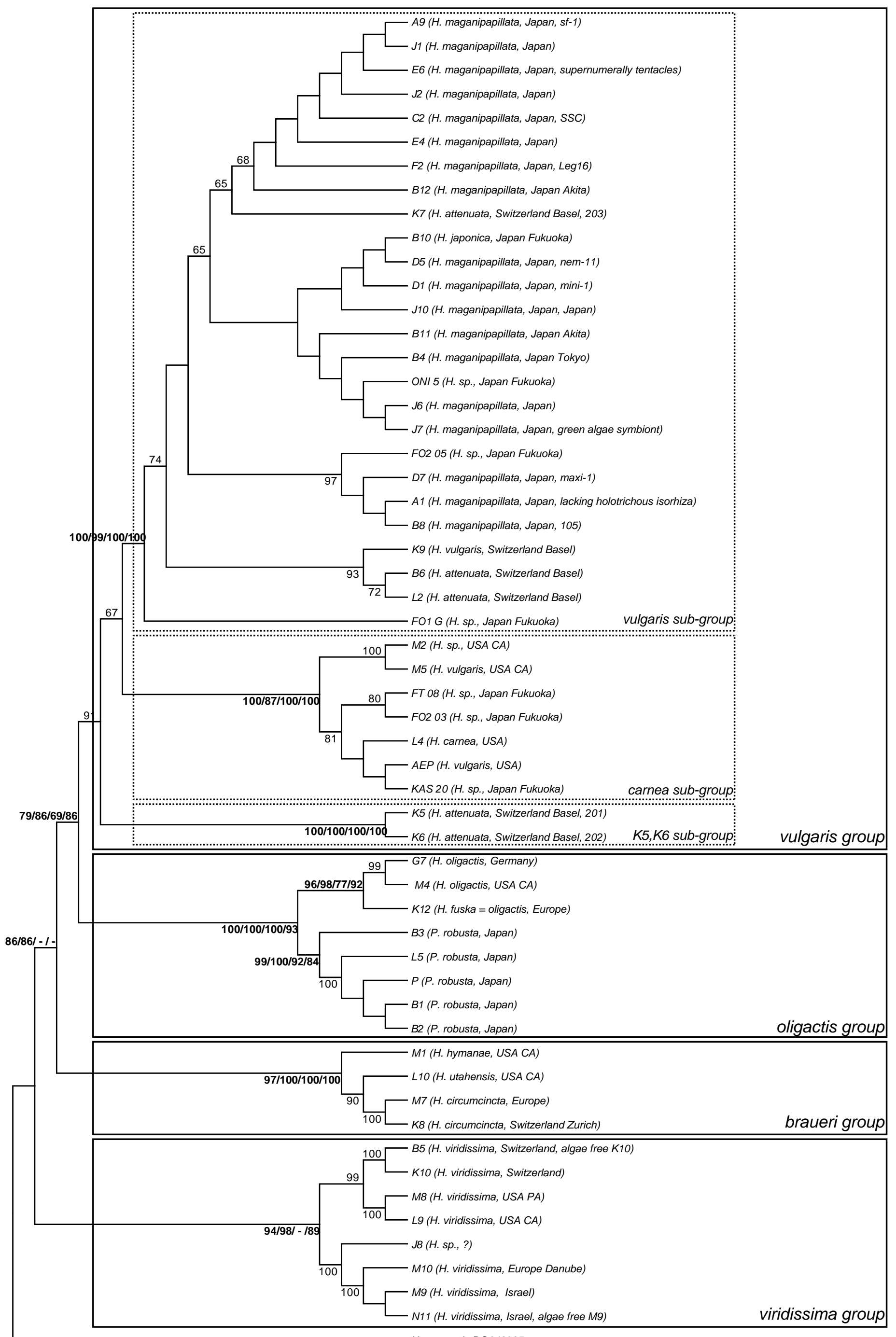




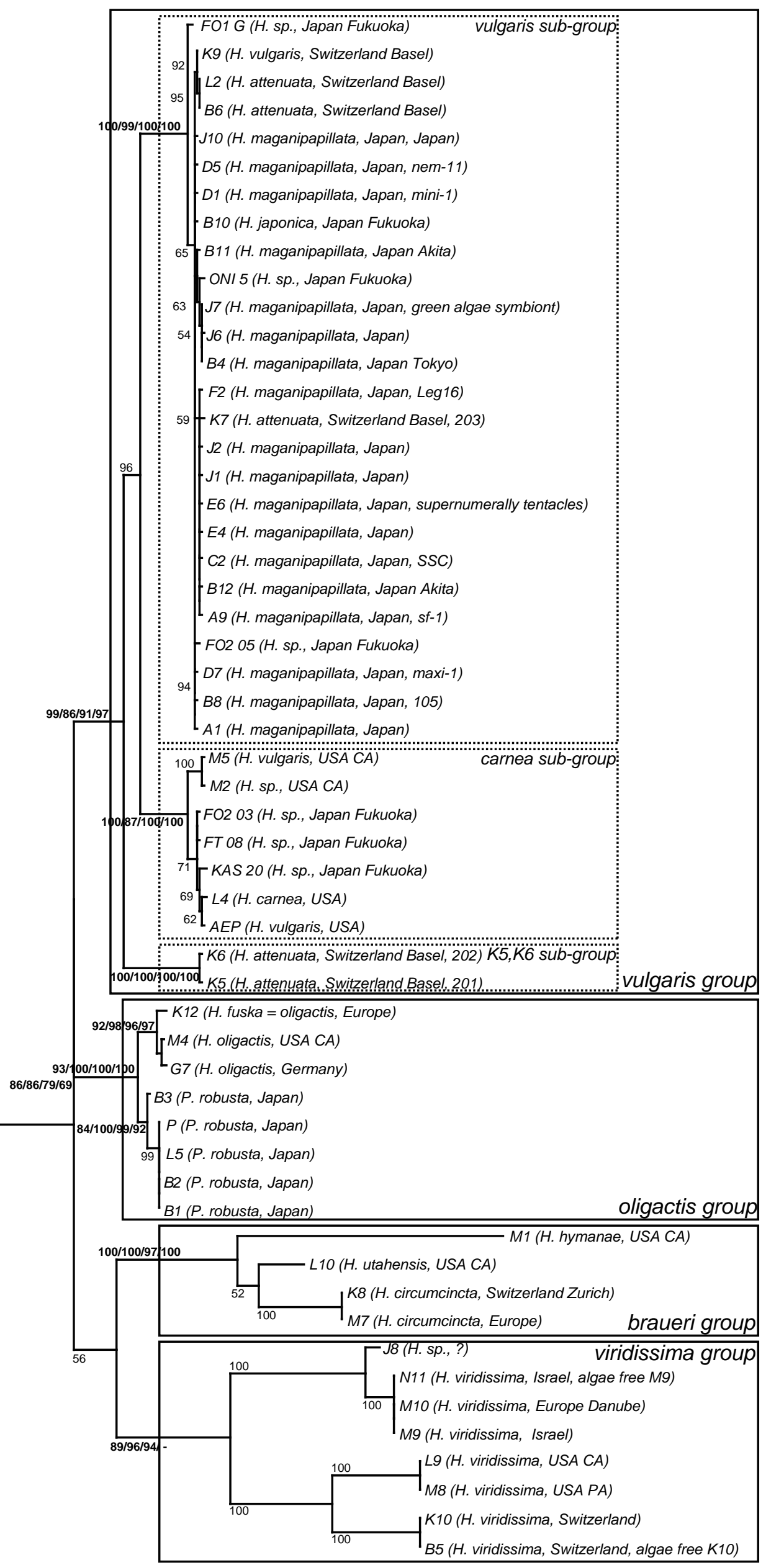

N. vectensis DQ643835 


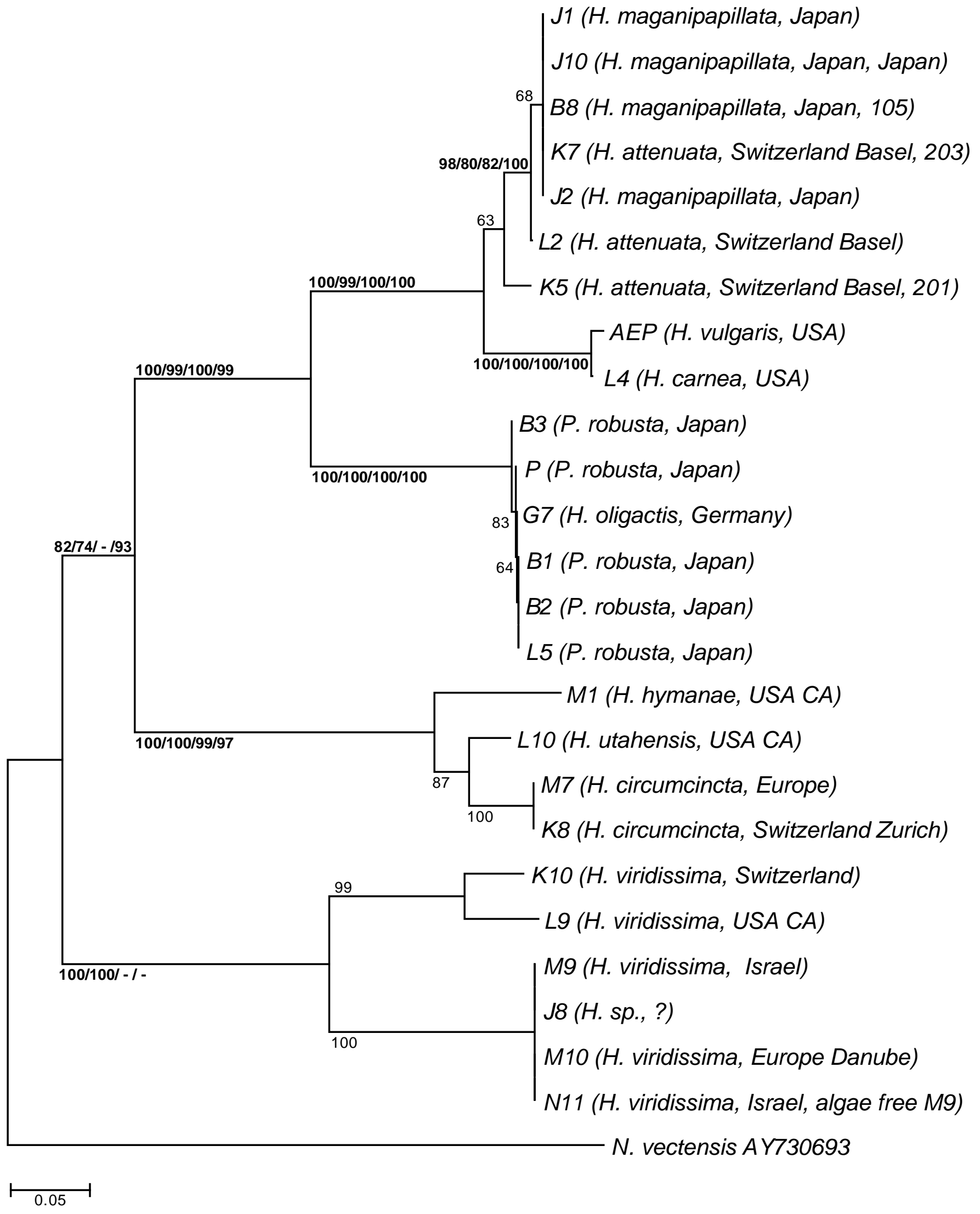




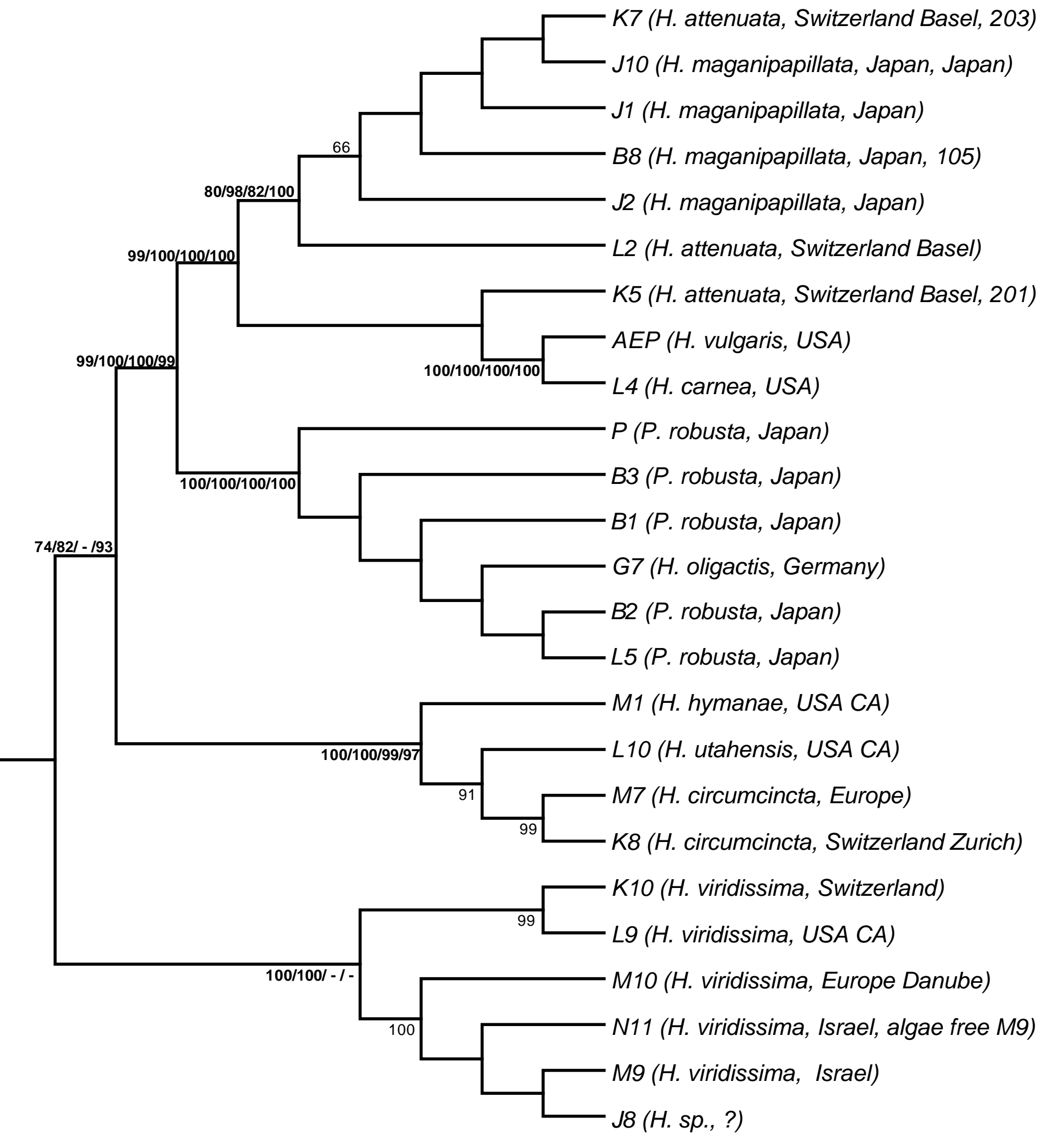

Nematostella AY730693 


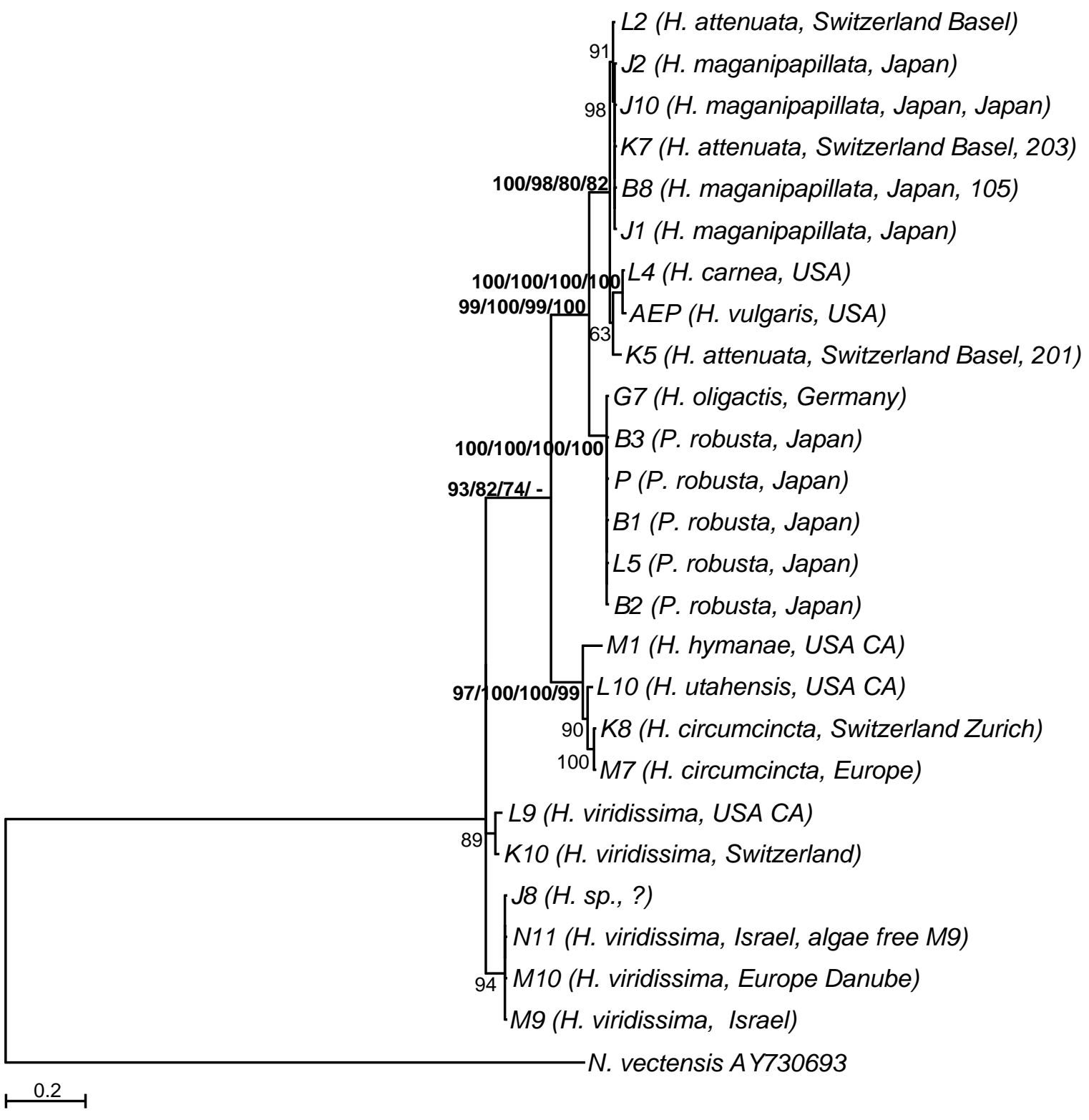




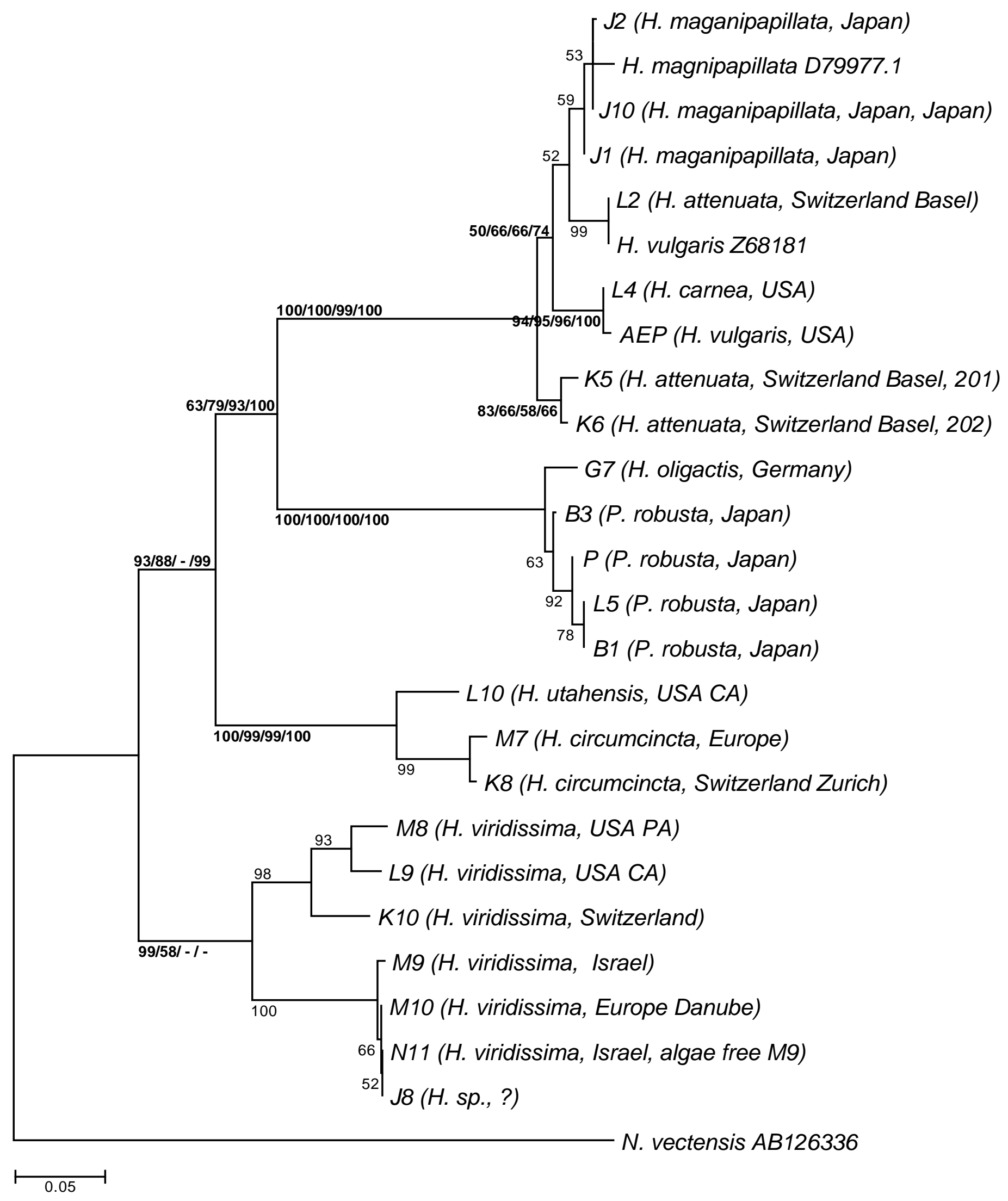




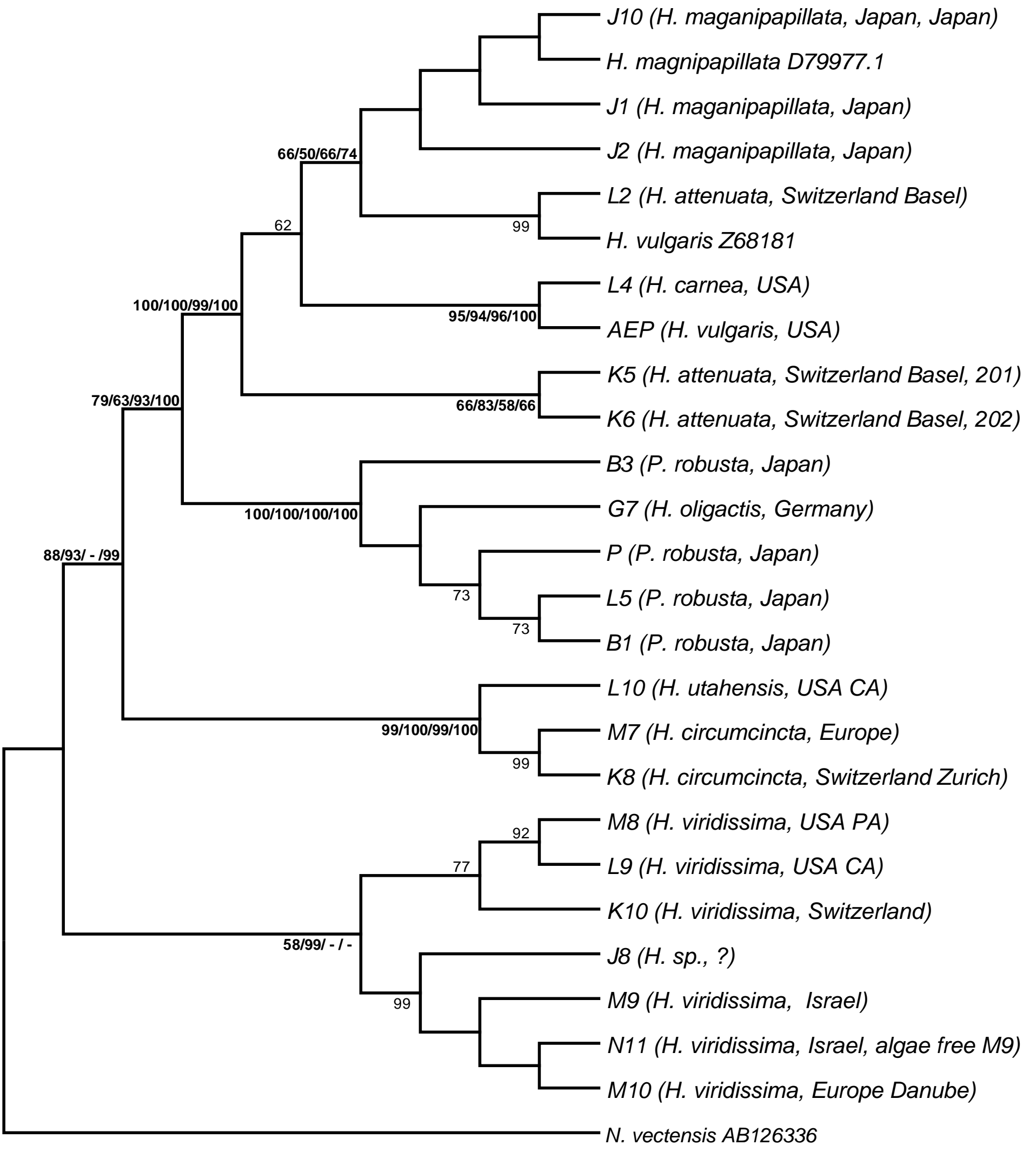




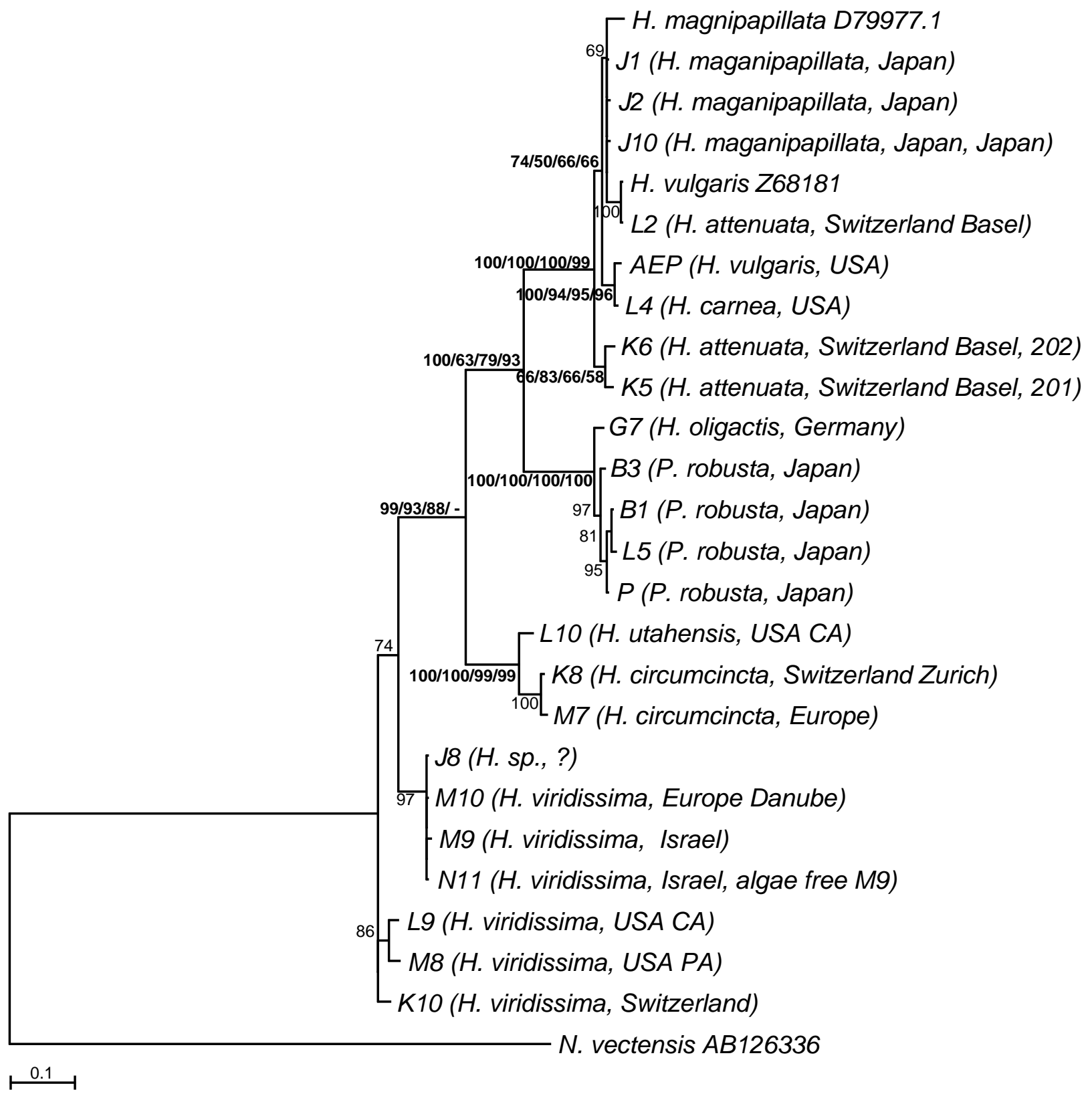


Supplemental Fig.4

Hydra utahensis (L10) Mt DNA Insert ORF (400 a.a.)

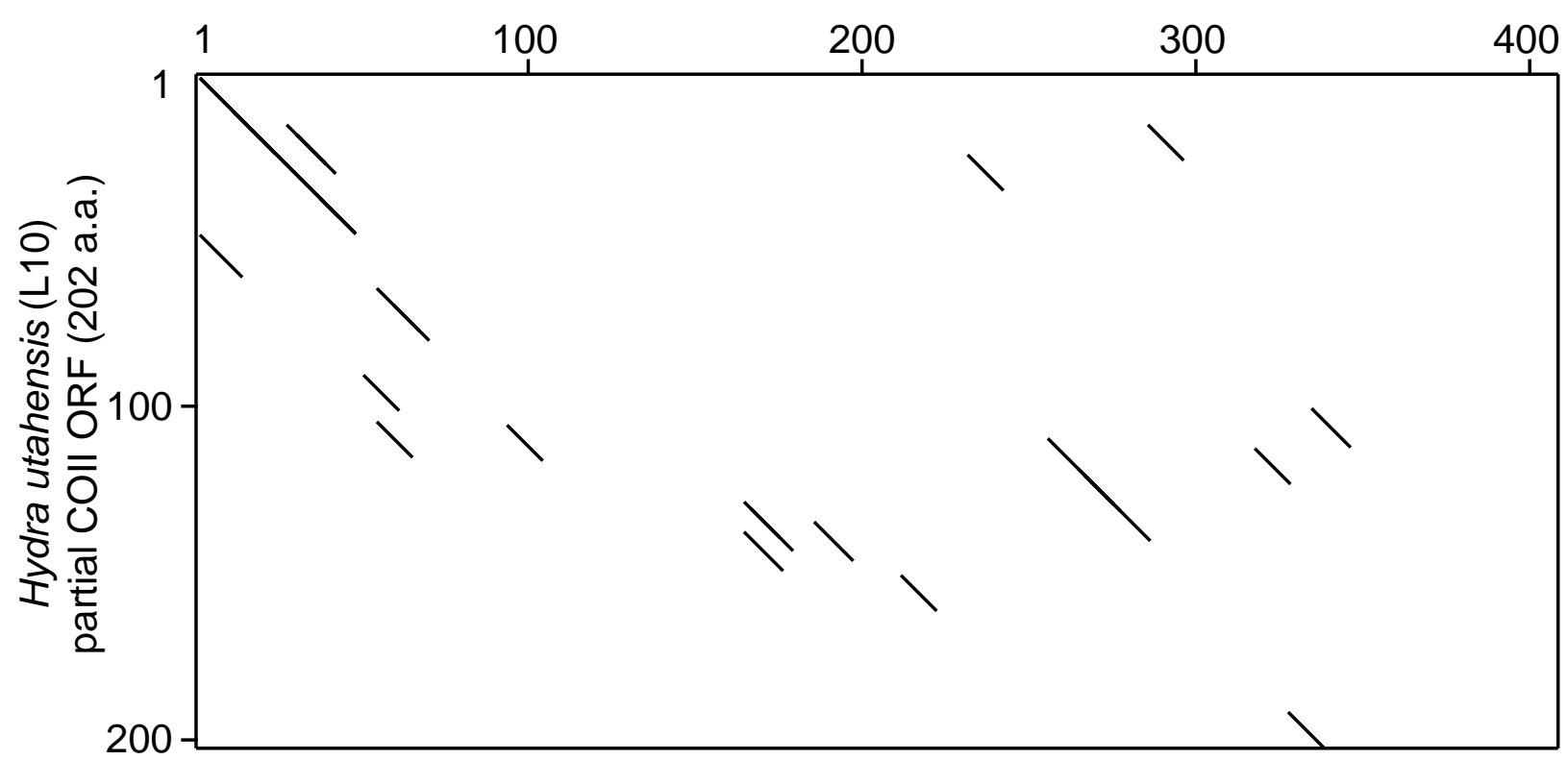

WindowSize: 10, Threshold: 21, ScoreMatrix: Blosum62 